\title{
Gravity-darkening exponents in semi-detached binary systems from their photometric observations: Part I
}

\author{
G. Djurašević ${ }^{1}$, H. Rovithis-Livaniou ${ }^{2}$, P. Rovithis ${ }^{3}$, N. Georgiades ${ }^{2}$, S. Erkapić ${ }^{1}$, and R. Pavlović ${ }^{1}$ \\ 1 Astronomical Observatory, Volgina 7, 11160 Belgrade, Yugoslavia and Isaac Newton Institute of Chile, Yugoslav branch \\ 2 Section of Astrophysics-Astronomy \& Mechanics, Dept. of Physics, Athens University, GR Zografos 157 84, Athens, Greece \\ e-mail: elivan@atlas.uoa.gr \\ ${ }^{3}$ Institute of Astronomy \& Astrophysics, National Observatory of Athens, PO Box 20048, 11810 Athens, Greece \\ e-mail: rovithis@astro.noa.gr
}

Received 24 June 2002 / Accepted 30 January 2003

\begin{abstract}
From the light curve analysis of several semi-detached close binary systems, the exponent of the gravity-darkening (GDE) for the Roche lobe filling components has been empirically estimated. The analysis, based on Roche geometry, has been made using the latest improved version of our computer programme. The present method of the light-curve analysis enables simultaneous estimation of the systems' parameters and the gravity-darkening exponents. The reliability of the method has been confirmed by its application to the artificial light curves obtained with a priori known parameters. Further tests with real observations have shown that in the case of well defined light curves the parameters of the system and the value of the gravitydarkening exponent can be reliably estimated. This first part of our analysis presents the results for 9 of the examined systems, that could be briefly summarised as follows:

1) For four of the systems, namely: ZZ Cru, RZ Dra, XZ Sgr and W UMi, there is a very good agreement between empirically estimated and theoretically predicted values for purely radiative and convective envelopes.

2) For the rest five, namely: TT Aur, V Pup, TV Cas, LT Her, and VV UMa, the estimated values of the gravity-darkening exponents were deduced to be larger than theory expects for purely radiative or convective envelopes, respectively. Moreover, it is worthwhile to mention that these values -although greater than theoretical predictions- are:

a) Smaller compared to those found by others.

b) In two cases, (TV Cas \& LT Her), they are normal if some kind of solar type activity (i.e. by employing the Roche model involving a spotted area on the surface of the secondary star) is assumed.

3) The large values derived for the two early type systems TT Aur \& V Pup, are very possibly connected with the effects of rotation laws, and in some degree with the large contribution of the radiative pressure in the total potential, leading by that to considerable deviations of the stellar surfaces from the assumed classical Roche geometry.

4) Finally, in the VV UMa case, the estimated value of the gravity-darkening exponent - although almost double than the expected for stars with convective envelopes - still it is low in comparison with that estimated by others who have analysed the same data. But, since for this particular binary the anomalous GDE is not supported by recent simultaneous uvby observations (Lazaro et al. 2002), the estimated higher value may be due to the old observational material used.
\end{abstract}

Key words. stars: general - binaries: close - stars: fundamental parameters - stars: general

\section{Introduction}

The emergent flux, $F$, of total radiation at any point over the surface of a rotationally or tidally distorted star in hydrostatic equilibrium varies proportionally to the local gravity acceleration $g$. This - known as the gravity-darkening law - is expressed as: $F \sim g^{\alpha}$, where $\alpha$ is the gravity-darkening coefficient. A consequence of this is that the star's effective temperature is: $T_{\text {eff }} \sim g^{\alpha / 4}$ or $T_{\text {eff }} \sim g^{\beta}$, where $\beta=\alpha / 4$ is known as the gravity-darkening exponent.

Send offprint requests to: G. Djurašević, e-mail: gdjurasevic@aob.bg.ac.yu
If the energy transfer in the sub-surface layers of the star is purely radiative, $\alpha=1$ or $\beta$ is equal to 0.25 (von Zeipel 1924), while for stars with convective envelopes, $\alpha=0.32$, or $\beta=0.08$ (Lucy 1967). According to Smith \& Worley (1974), the von Zeipel gravity-darkening law is strictly true only under certain conditions; while Webbing (1976), assuming a simple opacity law, pointed out that if the entropy is constant in an envelope, the values of the gravity-darkening exponent are compatible with those derived by Lucy (1967). Gravity-darkening has been studied by many authors (e.g. Sarna 1989; Anderson \& Shu 1977; Unno et al. 1994; Alencar \& Vaz 1997, etc.). More recently, in a series of papers Claret $(1995,1998,2000)$ has shown that a smooth transition is achieved between the 
two energy transport mechanisms. Furthermore, Claret pointed out that the gravity-darkening phenomenon is related not only with atmospheric parameters but also with the internal stellar structure and with details of the rotation law. These, as concerns very briefly "old" and "new" theoretical approaches to the gravity-darkening problem.

On the other hand, several observational approaches for the gravity-darkening determination have been made by various investigators so far. Among these first attempts, the works of Kitamura et al. (1957), Kopal (1968), Kopal \& Kitamura (1968), could be referred. Later, some other investigators tried to derive the values of the gravity-darkening exponents of specific stars, or groups of stars, using various methods (e.g. Budding \& Kopal 1970; Rafert \& Twigg 1980; Eaton et al. 1980; Hilditch 1981, etc.). Moreover, in a series of papers Kitamura \& Nakamura (1983, 1986, 1987a,b, 1988a,b, 1989), and Nakamura \& Kitamura (1992) deduced empirical values of it from an analysis of the photometric ellipticity effect in various types of close binaries.

In the present work, the gravity-darkening exponents - for the Roche lobe filling components - of some semi-detached eclipsing binaries have been estimated through their light curves analysis.

\section{Computational procedure}

Ordinary semi-detached systems are consisting of a main sequence primary component inside its Roche lobe and a subgiant (or giant) secondary star filling its corresponding lobe. To analyse this kind of systems, a modern computer programme (Djurašević et al. 1998) has been used. The programme is based on the Roche geometry and the principles arising from the paper by Wilson \& Devinney (1971). Moreover, it can be run choosing among three possibilities, in respect to the treatment of the radiation law: 1) simple black-body theory, 2) stellar atmosphere models by Carbon \& Gingerich (1969, CG) and 3 ) the Basel Stellar Library (BaSeL).

The present light-curves analysis was carried out using all three foregoing mentioned possibilities. This was necessary, since BaSeL models could not be used for all of the analyzed systems. To be more specific: in some particular cases observations were not given in the standard $U B V$ system, and in others the light curves were obtained in the Strömgren system. And as is known BaSeL fluxes do not exist for none of these cases. So, for these types of light curves we used only the black-body and $\mathrm{CG}$ approximations. The final choice of the approximation used in the light-curve analysis is made in such a way to keep the disagreement between different individual passbands solutions at the minimum. In the cases where the "corrected" BaSeL model flux distributions are used, solar chemical abundance is assumed for both of the components.

We have explored the "corrected" BaSeL model flux distributions, consistent with extent empirical calibrations (Lejeune et al. 1997, 1998). By solving the inverse problem of the light-curve analysis, the fluxes are calculated in each iteration for current values of temperatures and $\log g$, by interpolation for both of these quantities in the atmosphere tables, as an input, for a given metallicity $[\mathrm{Fe} / \mathrm{H}]$ of the close binary $(\mathrm{CB})$ components, where $[\mathrm{Fe} / \mathrm{H}]$ is the logarithmic metal abundance. The surface gravities can be derived very accurately from the masses and radii of $\mathrm{CB}$ stars, but the temperature determination is related to the assumed metallicity and strongly depends on photometric calibration. The two-dimensional flux interpolation in $T_{\text {eff }}$ and $\log g$ is based on the application of the bicubic spline interpolation (Press et al. 1992).

To achieve more reliable estimates of the model parameters in the programme for the light curves analysis, a quite dense coordinate grid, having $72 \times 144=10368$ elementary cells per each star, is used. The intensity and angular distribution of radiation of elementary cells are determined by stellar effective temperature, limb \& gravity-darkening and by the reflection effect.

Stellar sizes are described by the filling factors of the critical Roche lobes, $F_{\mathrm{h}} \& F_{\mathrm{c}}$, of the two components. The subscripts (h, c) are referred to the hotter and cooler component, respectively. For synchronous rotation of the components, these factors are expressed as the ratio of the stellar polar radii, $R_{\mathrm{h}, \mathrm{c}}$, and the corresponding polar radii of the critical Roche lobes, i.e., $F_{\mathrm{h}, \mathrm{c}}=R_{\mathrm{h}, \mathrm{c}} / R_{\text {Roche }_{\mathrm{h}, \mathrm{c}}}$.

For a successful application of the foregoing described model in the analysis of the observed light curves, the method proposed by Djurašević (1992) is used. By that optimum model parameters are obtained through the minimisation of $S=\Sigma(\mathrm{O}-\mathrm{C})^{2}$, where $\mathrm{O}-\mathrm{C}$ is the residual between the observed (LCO) and synthetic (LCC) light curves for a given orbital phase. The minimisation of $\Sigma$ is done in an iterative cycle of corrections of the model parameters by using the modified Marquardt's (1963) algorithm. In this way, the inverse-problem method provides the estimates of system's parameters and their standard errors.

Most of the authors that were dealing with the analysis of the limb-darkening effect (e.g., Díaz-Cordovés et al. 1995; Van Hamme 1993; Claret 1998) have pointed out that the linear approximation is not the best solution.

In our programme both limb-darkening laws were introduced, the linear and nonlinear (logarithmic and square root law). The nonlinear limb-darkening laws have been used in the present work to avoid the possible negative influence of the wrong evaluation of limb-darkening coefficients on other parameters in the inverse problem. Moreover, the option that allows the automatic choice of the square root approximation for stars hotter than $8000 \mathrm{~K}$, and the logarithmic law for stars with lower effective temperatures, (in agreement with Van Hamme's 1993 paper, whose tables we used), exists in our programme, too.

The approximations can be written in the form:

$I_{\lambda}(\mu)=I_{\lambda}(1)\left(1-a_{\lambda}(1-\mu)-b_{\lambda} \mu \ln \mu\right)$

- logarithmic law;

$I_{\lambda}(\mu)=I_{\lambda}(1)\left(1-c_{\lambda}(1-\mu)-d_{\lambda}(1-\sqrt{\mu})\right)$

- root square law;

where $a_{\lambda}$ and $b_{\lambda}$ are the logarithmic limb-darkening passband specific coefficients, $c_{\lambda}$ and $d_{\lambda}$ the root square ones and $\mu=$ $\cos \gamma . I_{\lambda}(1)$ is the passband specific intensity at the center of 
the stellar disc, and $\gamma$ is the angle between the line of sight and the emergent flux. The values of the limb-darkening coefficients are derived from the current values of the stellar effective temperature $T_{\text {eff }}$ and surface gravity $\log g$ in each iteration, by interpolation for both of these quantities in the Van Hamme (1993) tables. This was achieved by bicubic spline or bi-linear interpolation (Press et al. 1992).

Since the primary component is well deep inside its Roche lobe, this star's shape is very close to that of a sphere. In such a case, and following Rucinski (1969) and Rafert \& Twigg (1980), the gravity-darkening exponents of these stars, $\beta$, are set at their theoretical values. That is, $\beta=0.25$ for stars in hydrostatic and radiative equilibrium (von Zeipel 1924), and $\beta=0.08$ for those with convective envelopes (Lucy 1967). Concerning their albedos, $A$, the values of $A=1.0$, and $A=0.5$ were given, respectively.

To check the reliability of the method of simultaneous estimation of the system's parameters and gravity-darkening exponent we performed a number of numerical tests where we used artificial synthetic light curves obtained with known parameters. The tests proved that the inverse-problem method has successfully found the true parameters used to produce the artificial light curves even with coarse initial parameters of the system. This suggests that the method can be used in the analysis of real observations. The reliability of the estimated parameters depends on the quality of photometric observations.

This forced us to pay special attention to the choice of the systems. To increase the estimation credibility we've chosen only systems with well defined light curves.

The errors of the parameter estimates arise from the nonlinear least-squares method, on which the inverse-problem method is based. The uncertainties of these parameters may be larger than we estimated. Our estimate of the accuracy in the determination of these parameters is based on the influence of formal errors arising from the nonlinear method of the light-curve analysis. Having in mind the errors of the input parameters of the model, which are treated as fixed in the inverse-problem method, the real errors of the parameters will definitely be larger (approximately 2-3 times). The main contribution comes from the error in effective temperature of the primary fixed on the basis of its spectral type, which means with a relatively high uncertainty. Thus, the estimated error of the secondary component's temperature is significantly larger than the tabular values obtained under the assumption that the temperature of the primary is accurate. The errors in the estimates of the stellar radii are included (through the filling factors), while the errors in the masses are not formally used (the mass ratio is treated as fixed), but certainly they contribute in real accuracy of the system parameter estimation. Because of all these, the real error bars in the GDE estimates are larger than the values given in the tables. Based on the available data, we cannot give a real estimate of the total accuracy of the system parameters.

\section{Results of the light-curve analysis}

In this first part of our work, as was already mentioned, we present the results derived from the light-curve analysis of nine

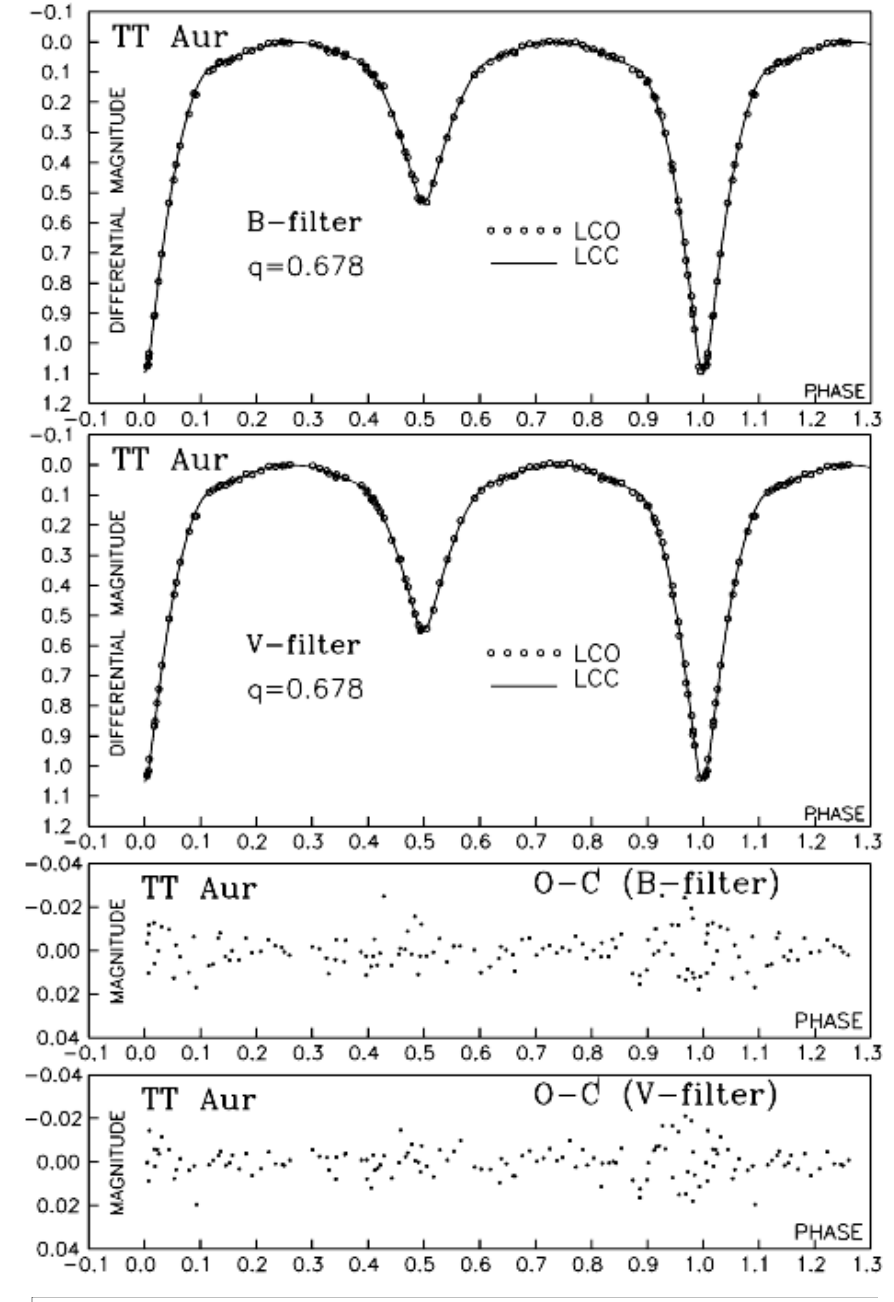

TT Aur
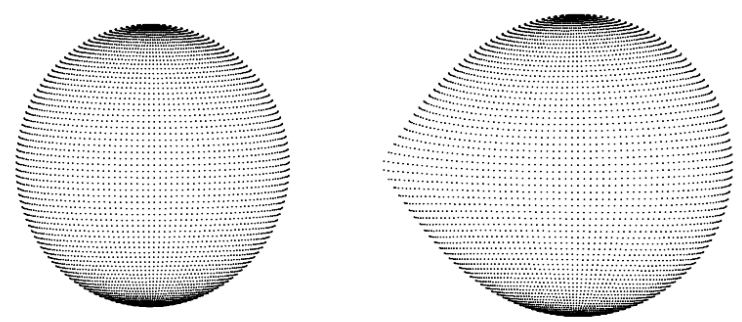

PHASE $=0.25$

Fig. 1. Observed (LCO) and final synthetic (LCC) light curves of TT Aur with final O-C residuals obtained by analysing the light curves and the view of the systems at orbital phase 0.25 , obtained with the parameters estimated by analysing observations.

semi-detached eclipsing binaries. A summary for each system follows, starting from the two early-type systems.

\subsection{TT Aurigae $\left(P \sim 1^{\mathrm{d}} .333\right)$}

The massive semi-detached system TT Aur contains two B-type stars. In our analysis we used the same observations and the same input physical parameters as Kitamura \& Nakamura (1987b) to be able to compare our results with their. So, the 
Table 1. Results of the analysis of TT Aur and V Pup light curves obtained by solving the inverse problem for the Roche model. Gravitydarkening exponent of the cooler secondary component $\left(\beta_{\mathrm{c}}\right)$ is a free parameter.

\begin{tabular}{|c|c|c|c|c|c|}
\hline $\begin{array}{l}\text { CB system } \\
\text { quantity }\end{array}$ & $\begin{array}{l}\text { TT Aur } \\
B-\text { filter }\end{array}$ & $\begin{array}{l}\text { TT Aur } \\
V \text {-filter }\end{array}$ & $\begin{array}{l}\text { V Pup } \\
v \text {-filter }\end{array}$ & $\begin{array}{l}\text { V Pup } \\
b-\text { filter }\end{array}$ & $\begin{array}{l}\text { V Pup } \\
y \text {-filter }\end{array}$ \\
\hline$n$ & 113 & 113 & 1281 & 1281 & 1281 \\
\hline$\Sigma(\mathrm{O}-\mathrm{C})^{2}$ & 0.0079 & 0.0066 & 0.0480 & 0.0458 & 0.0590 \\
\hline$\sigma$ & 0.0084 & 0.0075 & 0.0061 & 0.0060 & 0.0068 \\
\hline$q=m_{\mathrm{c}} / m_{\mathrm{h}}$ & 0.678 & & 0.53 & & \\
\hline$T_{\mathrm{h}}$ & 23100 & & 28200 & & \\
\hline$\beta_{\mathrm{h}}$ & 0.25 & & 0.25 & & \\
\hline$A_{\mathrm{h}}=A_{\mathrm{c}}$ & 1.0 & & 1.0 & & \\
\hline$f_{\mathrm{h}}=f_{\mathrm{c}}$ & 1.0 & & 1.0 & & \\
\hline$T_{\mathrm{c}}$ & $17720 \pm 48$ & $17516 \pm 48$ & $25816 \pm 30$ & $25908 \pm 31$ & $25820 \pm 37$ \\
\hline$F_{\mathrm{h}}$ & $0.778 \pm 0.007$ & $0.782 \pm 0.007$ & $0.928 \pm 0.001$ & $0.929 \pm 0.001$ & $0.929 \pm 0.001$ \\
\hline$F_{\mathrm{c}}$ & $1.000 \pm 0.001$ & $1.000 \pm 0.001$ & $1.000 \pm 0.001$ & $1.000 \pm 0.001$ & $1.000 \pm 0.001$ \\
\hline$i\left[^{\circ}\right]$ & $86.36 \pm 0.09$ & $86.39 \pm 0.09$ & $78.08 \pm 0.03$ & $78.01 \pm 0.03$ & $78.02 \pm 0.03$ \\
\hline$\beta_{\mathrm{c}}$ & $0.36 \pm 0.01$ & $0.38 \pm 0.01$ & $0.380 \pm 0.006$ & $0.388 \pm 0.006$ & $0.382 \pm 0.007$ \\
\hline$c_{\mathrm{h}}$ & -0.109 & -0.093 & -0.118 & -0.120 & -0.120 \\
\hline$d_{\mathrm{h}}$ & 0.672 & 0.580 & 0.680 & 0.652 & 0.605 \\
\hline$c_{\mathrm{c}}$ & -0.084 & -0.070 & -0.112 & -0.114 & -0.115 \\
\hline$d_{\mathrm{c}}$ & 0.699 & 0.595 & 0.684 & 0.655 & 0.607 \\
\hline$\Omega_{\mathrm{h}}$ & 3.955 & 3.937 & 3.129 & 3.126 & 3.125 \\
\hline$\Omega_{\mathrm{c}}$ & 3.204 & 3.205 & 2.933 & 2.933 & 2.933 \\
\hline$R_{\mathrm{h}}[D=1]$ & 0.302 & 0.304 & 0.380 & 0.380 & 0.380 \\
\hline$R_{\mathrm{c}}[D=1]$ & 0.324 & 0.324 & 0.304 & 0.304 & 0.304 \\
\hline$L_{\mathrm{h}} /\left(L_{\mathrm{h}}+L_{\mathrm{c}}\right)$ & 0.571 & 0.563 & 0.637 & 0.635 & 0.634 \\
\hline $\mathcal{M}_{\mathrm{h}}\left[M_{\odot}\right]$ & 6.80 & 6.80 & 14.92 & 14.94 & 14.93 \\
\hline $\mathcal{M}_{\mathrm{c}}\left[M_{\odot}\right]$ & 4.61 & 4.61 & 7.91 & 7.92 & 7.92 \\
\hline $\mathcal{R}_{\mathrm{h}}\left[R_{\odot}\right]$ & 3.56 & 3.58 & 6.10 & 6.11 & 6.11 \\
\hline $\mathcal{R}_{\mathrm{c}}\left[R_{\odot}\right]$ & 3.97 & 3.96 & 4.98 & 4.98 & 4.98 \\
\hline $\log g_{\mathrm{h}}$ & 4.17 & 4.16 & 4.04 & 4.04 & 4.04 \\
\hline $\log g_{\mathrm{c}}$ & 3.90 & 3.90 & 3.94 & 3.94 & 3.94 \\
\hline$M_{\mathrm{bol}}^{\mathrm{h}}$ & -3.99 & -4.00 & -6.02 & -6.03 & -6.03 \\
\hline$M_{\mathrm{bol}}^{\mathrm{c}}$ & -3.07 & -3.02 & -5.20 & -5.22 & -5.20 \\
\hline$a_{\mathrm{orb}}\left[R_{\odot}\right]$ & 11.46 & 11.46 & 15.31 & 15.31 & 15.31 \\
\hline
\end{tabular}

TT Aur: Black-body approximation of stellar atmosphere.

V Pup: Carbon \& Gingerich (1969) approximation of stellar atmosphere.

Note: $n$ - number of observations, $\Sigma(\mathrm{O}-\mathrm{C})^{2}-$ final sum of squares of residuals between observed (LCO) and synthetic (LCC) light curves; $\sigma$ - standard deviation of the observations; $q=m_{\mathrm{c}} / m_{\mathrm{h}}$ - mass ratio of the components; $T_{\mathrm{h}}$ - temperature of the hotter component; $\beta_{\mathrm{h}}-$ gravitydarkening exponent of the hotter component; $A_{\mathrm{h}}=A_{\mathrm{c}}=1.0$ - albedo coefficients of the components; $f_{\mathrm{h}}=f_{\mathrm{c}}=1.00-$ nonsynchronous rotation coefficients of the components, $T_{\mathrm{c}}$ - temperature of the cooler component; $F_{\mathrm{h}, \mathrm{c}}-$ filling factors for critical Roche lobes of the hotter primary (h) and cooler secondary (c); $i$ - orbit inclination (in arc degrees); $\beta_{\mathrm{c}}$ - gravity-darkening exponent of the cooler secondary component; $c_{\mathrm{h}, \mathrm{c}}, d_{\mathrm{h}, \mathrm{c}}$ - nonlinear limb-darkening coefficients of the components (square root law); $\Omega_{\mathrm{h}, \mathrm{c}}$ - dimensionless surface potentials of the components; $R_{\mathrm{h}, \mathrm{c}}-$ polar radii of the components in units of the distance between the components centers; $L_{\mathrm{h}} /\left(L_{\mathrm{h}}+L_{\mathrm{c}}\right)-$ luminosity of the hotter star; $\mathcal{M}_{\mathrm{h}, \mathrm{c}}\left[M_{\odot}\right]$ - masses of stars in solar units; $\mathcal{R}_{\mathrm{h}, \mathrm{c}}\left[R_{\odot}\right]$ - mean radii of stars in solar units; $\log g_{\mathrm{h}, \mathrm{c}}-\operatorname{logarithm}($ base 10$)$ of the mean surface acceleration (effective gravity) for components, $M_{\mathrm{bol}}^{\mathrm{h}, \mathrm{c}}-$ absolute bolometric magnitudes of stars; $a_{\mathrm{orb}}\left[R_{\odot}\right]-$ orbital semi-major axis in units of the solar radius.

light curves in $B\left(\lambda_{\mathrm{eff}}=433 \mathrm{~nm}\right)$ and $V\left(\lambda_{\mathrm{eff}}=547 \mathrm{~nm}\right)$ filters for which normal points were published in (Wachmann et al. 1986), were used. The analysis was made assuming the mass-ratio of the components equal to $q=m_{\mathrm{c}} / m_{\mathrm{h}}=0.678$ (Wachmann et al. 1986). Based on the spectral type of the components, B2 V and B4 (Kitamura \& Nakamura 1987b), the value of $T_{\mathrm{h}}=23100 \mathrm{~K}$ was assumed as the temperature of the primary star. The gravity-darkening exponent of the primary was fixed to $\beta_{\mathrm{h}}=0.25$, and as concerns the albedos of the components the value of $A_{\mathrm{h}}=A_{\mathrm{c}}=1.0$ was adopted for both 
of them. Other parameters were estimated in the light-curve analysis. In this case the simple black-body approximation for the stellar atmosphere provides better mutual agreement of the individual $B$ and $V$ solutions than $\mathrm{CG}$ approximation. BaSeL approximation is not appropriate here, because no standard filters were used to get the observations.

The parameters derived from the light-curve analysis are listed in Table 1. The first three rows of the table present the number of observations $n$, the final sum of squares of residuals between observed (LCO) and synthetic (LCC) light curves

$\sum_{i=1}^{n}\left(\mathrm{O}_{i}-\mathrm{C}_{i}\right)^{2}$,

and the standard deviation of the observations

$\sigma=\sqrt{\frac{\sum_{i=1}^{n}\left(\mathrm{O}_{i}-\mathrm{C}_{i}\right)^{2}}{(n-1)}}$.

A mean value $\beta_{c} \sim 0.37$ for the gravity-darkening exponent of the secondary star is derived from our solution of $B \& V$ curves of TT Aur. This value is 1.48 times higher than that expected for stars in hydrostatic and radiative equilibrium. With the same observational data by applying a different method Kitamura \& Nakamura (1987b) found a mean value $\alpha_{\mathrm{c}}=4 \times \beta_{\mathrm{c}} \sim 4.14$ for this system, which is about 2.8 times higher than the value estimated by us in the present paper. Having in mind the use of identical light curves with the same initial parameters of the system, the cause of this discrepancy should be find in the method used.

Figure 1 gives a graphic presentation of these results, where the optimum fit of the observed light curves (LCO) to the synthetic ones (LCC) is shown. The $\mathrm{O}-\mathrm{C}$ residuals between the observed (LCO) and optimum synthetic (LCC) light curves are given for each individual light curve, too. Finally this panel shows the view of Roche model of the system, obtained with the parameters estimated by analysing the light curves. Using such plots, one sees how a CB system would look at a certain orbital phase.

\section{2. $V$ Puppis $(P \sim 1.454)$}

In the analysis of the massive B-type semi-detached system V Pup, the vby light curves by Clausen et al. (1983) in Strömgren system were used. The light-curve analysis was made with the mass-ratio of the components fixed to $q_{\mathrm{sp}}=$ $m_{\mathrm{c}} / m_{\mathrm{h}}=0.53$ (Andersen et al. 1983). On the basis of the spectral type of the components, B1 V and B3 (Kitamura \& Nakamura $1987 \mathrm{~b}$ ), the value $T_{\mathrm{h}}=28200 \mathrm{~K}$ was adopted as the primary's star temperature. The gravity-darkening exponent of the primary was fixed to the value $\beta_{\mathrm{h}}=0.25$ and the values $A_{\mathrm{h}}=A_{\mathrm{c}}=1.0$ were used as the albedos of the components. The best agreement between individual $v, b$ and $y$ solutions found from the light-curve analysis was obtained by applying the CG stellar atmosphere models.

Table 1 shows the results of our analysis, that are graphically presented in Fig. 2 (left). The value of the gravitydarkening exponent of the secondary is $\beta_{\mathrm{c}} \sim 0.383$, which is approximately 1.5 times beyond the value expected for stars in hydrostatic and radiative equilibrium $(\beta=0.25)$. For this system Kitamura \& Nakamura (1987b) found $\alpha_{\mathrm{c}}=4 \times \beta_{\mathrm{c}}=5.44$ which is about 3.6 times higher than our value. Since the same observational material and the same input parameters have been used, that yield to different results, we think that the cause of the derived differences comes from the essentially different method used in the analysis of the light curves.

\section{3. $X Z$ Sagitarii $\left(P \sim 3^{\mathrm{d}} \cdot 2755\right)$}

To analyse this system we used the $U B V$ light curves by Kappelmann \& Walter (1979). Again, the light-curve analysis was made with the fixed mass-ratio of the components, $q_{\mathrm{sp}}=m_{\mathrm{c}} / m_{\mathrm{h}}=0.144$ (Smak 1965). The spectral type of the components in the system, A3 + G5 (Knipe 1974), gave us the temperature of the primary, $T_{\mathrm{h}}=8720 \mathrm{~K}$, which we adopted. The gravity-darkening exponent of the primary was fixed to the value $\beta_{\mathrm{h}}=0.25$, while the values $A_{\mathrm{h}}=1.0$ and $A_{\mathrm{c}}=0.5$ were adopted for the two components' albedos.

Table 2 presents the results of the light-curve analysis of this system, and the graphic presentation is given in Fig. 2 (right). BaSeL approximation provided better agreement between the individual $U B V$ passband solutions than the blackbody or $\mathrm{CG}$ approximation. For the gravity-darkening exponent of the secondary we found the value $\beta_{\mathrm{c}} \sim 0.08$, which is expected for stars with convective envelopes.

\subsection{VV Ursae Majoris $(P \sim 0 \mathrm{~d} 687)$}

The two colour ( $B$ and $V$ ) curves of Broglia \& Conconi (1977) were used for this system. The initial values of the parameters of the model were taken according to the results of the analysis presented in the paper by Rafert (1990). The temperature of the primary was taken to be $T_{\mathrm{h}}=9555 \mathrm{~K}$, as suggested by Hill et al.'s (1975) spectral classification of A2 V. Based on the spectral type of the components $(\mathrm{A} 2 \mathrm{~V}+[\mathrm{G} 8])$ the gravitydarkening exponent of the primary was fixed to $\beta_{\mathrm{h}}=0.25$, and the albedos of the components were taken as equal to $A_{\mathrm{h}}=1.0$ and $A_{\mathrm{c}}=0.5$. The parameters of the system were estimated in the light-curve analysis with the fixed mass-ratio of $q=m_{\mathrm{c}} / m_{\mathrm{h}}=0.298$ (Rafert 1990). In this case we used "corrected" BaSeL model flux distributions with the assumed solar chemical abundance for the components of the system. This approximation provided much better agreement between the individual $B$ and $V$ solutions than the simple black-body theory or CG stellar atmosphere models.

The results of the light-curve analysis are given in Table 2 and their graphical presentation is given in Fig. 5 (left). For the gravity-darkening exponent of the secondary we found the value $\beta_{\mathrm{c}} \sim 0.175$, which is approximately 2.2 times higher than the value expected for stars with convective envelopes $(\beta \sim$ 0.08). By applying a different model and method Kitamura \& Nakamura (1987b) found $\alpha_{\mathrm{c}}=4 \times \beta_{\mathrm{c}}=3.78$ for this system, which is about 5.5 times higher than our value. 

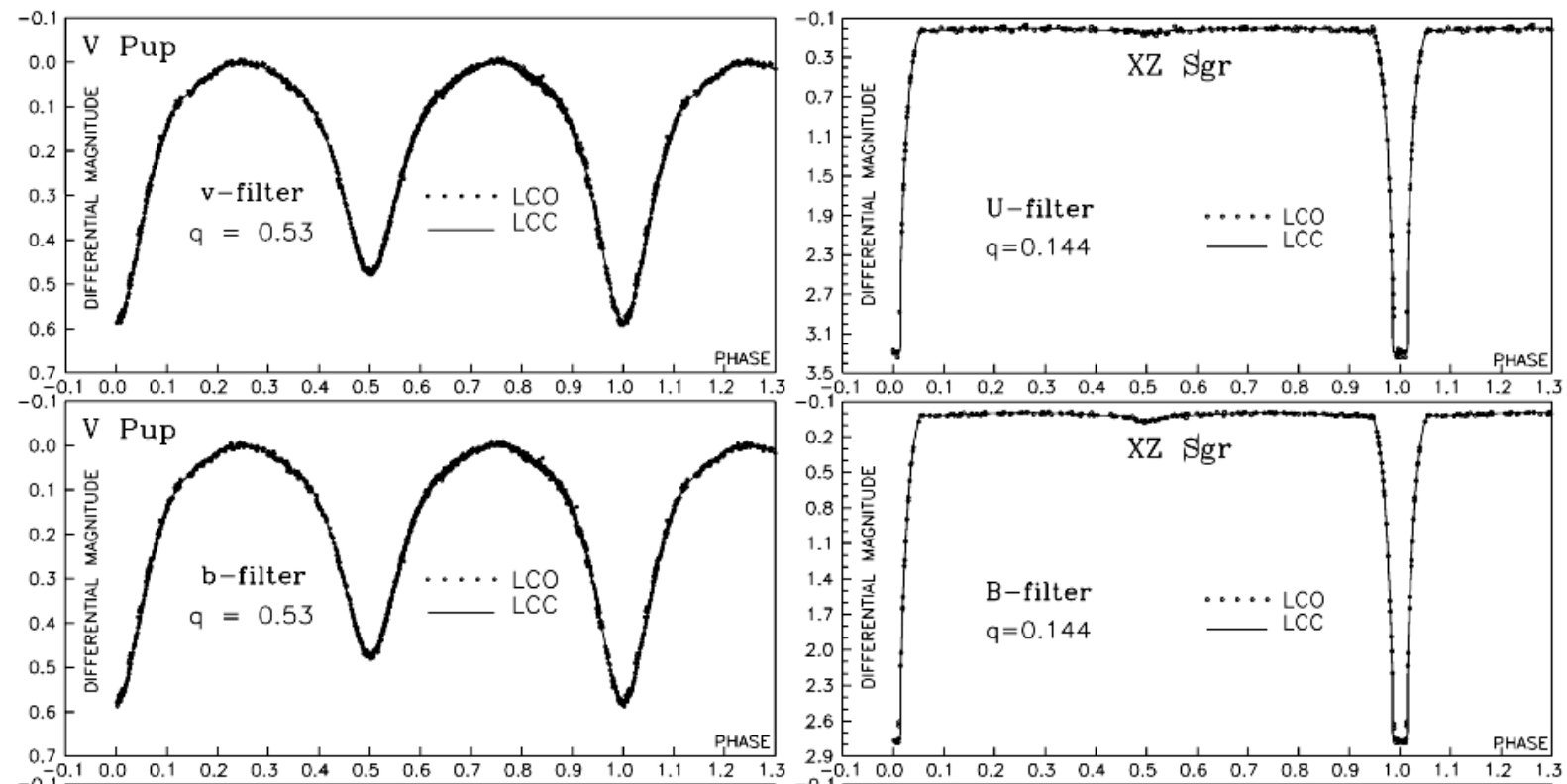

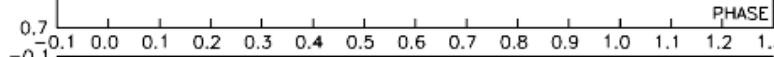
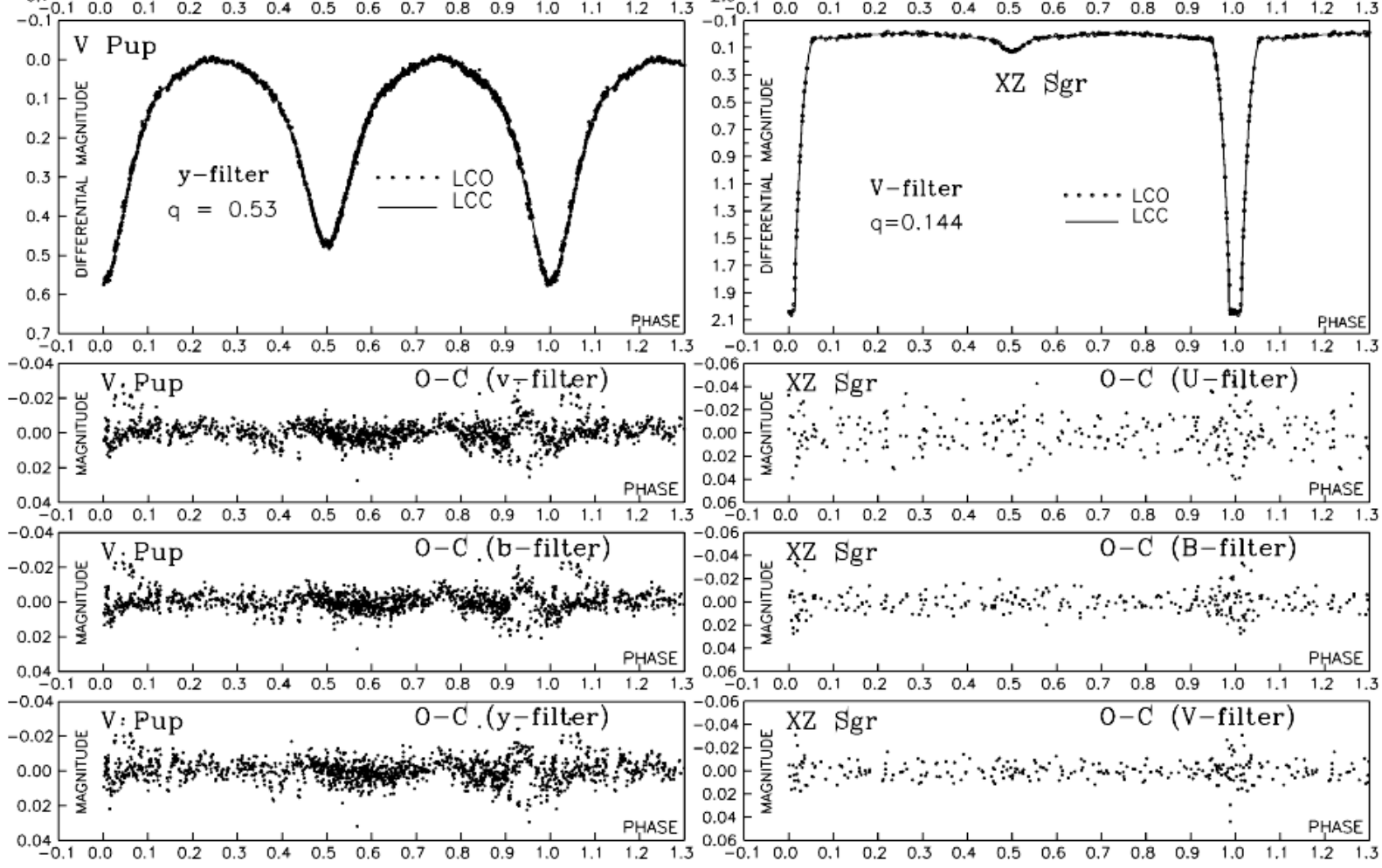

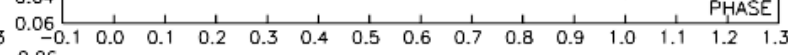
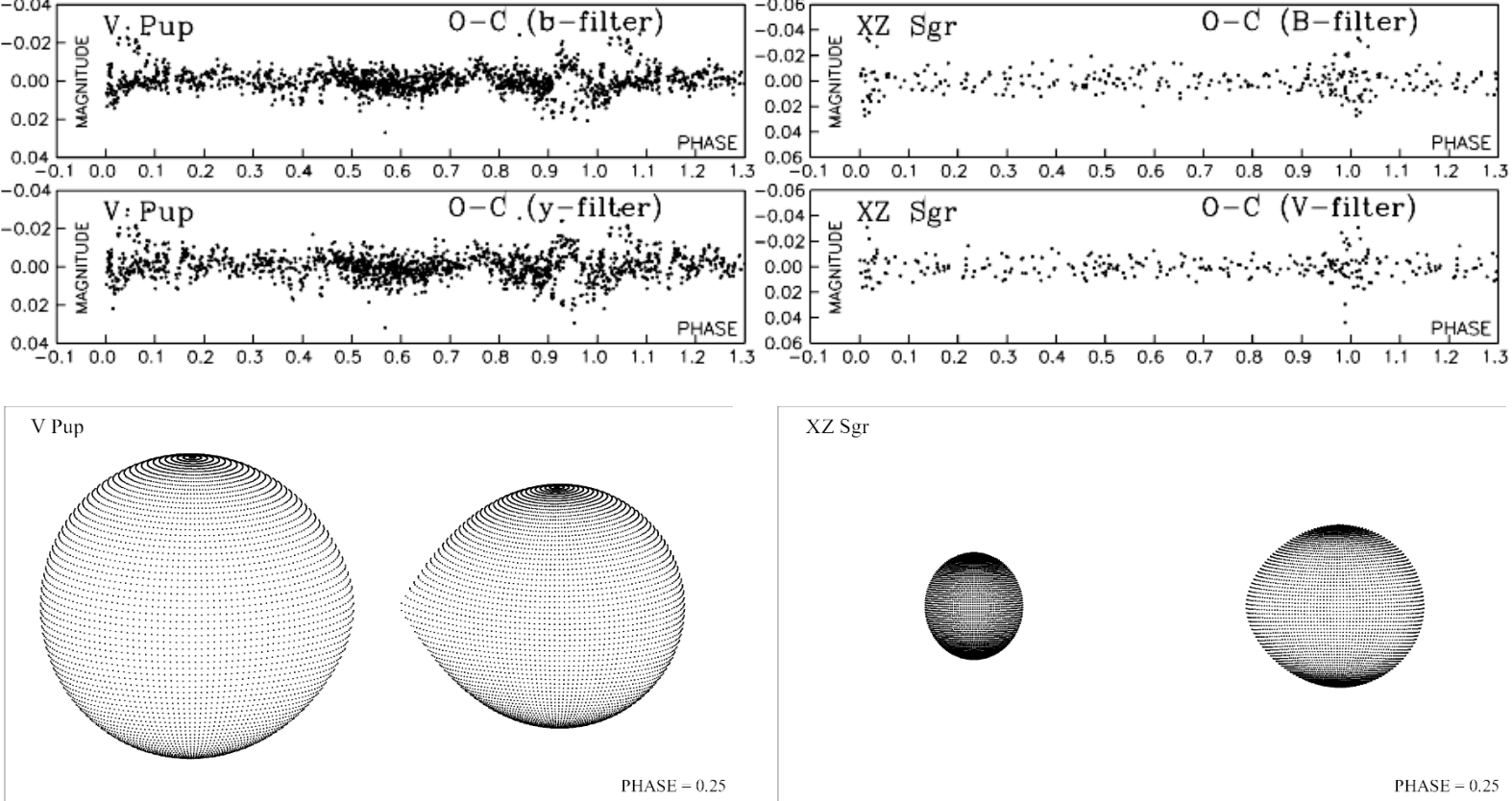

Fig. 2. Observed (LCO) and final synthetic (LCC) light curves of V Pup and XZ Sgr with final O-C residuals obtained by analysing the light curves and the view of the systems at orbital phase 0.25 , obtained with the parameters estimated by analysing observations. 
Table 2. Results of the analysis of XZ Sgr, VV UMa and RZ Dra light curves obtained by solving the inverse problem for the Roche model. Gravity-darkening exponent of the cooler secondary component $\left(\beta_{\mathrm{c}}\right)$ is a free parameter.

\begin{tabular}{|c|c|c|c|c|c|c|c|}
\hline $\begin{array}{l}\text { CB system } \\
\text { quantity }\end{array}$ & $\begin{array}{l}\text { XZ Sgr } \\
U-\text { filter }\end{array}$ & $\begin{array}{l}\text { XZ Sgr } \\
B-\text { filter }\end{array}$ & $\begin{array}{l}\text { XZ Sgr } \\
V-\text { filter }\end{array}$ & $\begin{array}{l}\text { VV UMa } \\
B \text {-filter }\end{array}$ & $\begin{array}{l}\text { VV UMa } \\
V \text {-filter }\end{array}$ & $\begin{array}{l}\text { RZ Dra } \\
B-\text { filter }\end{array}$ & $\begin{array}{l}\text { RZ Dra } \\
V-\text { filter }\end{array}$ \\
\hline$n$ & 212 & 217 & 219 & 771 & 845 & 45 & 46 \\
\hline$\Sigma(\mathrm{O}-\mathrm{C})^{2}$ & 0.0507 & 0.0175 & 0.0151 & 0.7720 & 0.0666 & 0.0183 & 0.0125 \\
\hline$\sigma$ & 0.0155 & 0.0090 & 0.0083 & 0.0101 & 0.0089 & 0.0204 & 0.0167 \\
\hline$q=m_{\mathrm{c}} / m_{\mathrm{h}}$ & 0.144 & & & 0.298 & & 0.445 & \\
\hline$T_{\mathrm{h}}$ & 8720 & & & 9555 & & 8150 & \\
\hline$\beta_{\mathrm{h}}$ & 0.25 & & & 0.25 & & 0.25 & \\
\hline$A_{\mathrm{h}}$ & 1.0 & & & 1.0 & & 1.0 & \\
\hline$A_{\mathrm{c}}$ & 0.5 & & & 0.5 & & 0.5 & \\
\hline$f_{\mathrm{h}}=f_{\mathrm{c}}$ & 1.0 & & & 1.0 & & 1.0 & \\
\hline$T_{\mathrm{c}}$ & $5146 \pm 27$ & $5101 \pm 20$ & $5043 \pm 27$ & $5755 \pm 27$ & $5680 \pm 18$ & $5372 \pm 57$ & $5433 \pm 37$ \\
\hline$F_{\mathrm{h}}$ & $0.262 \pm 0.001$ & $0.261 \pm 0.001$ & $0.261 \pm 0.001$ & $0.765 \pm 0.001$ & $0.764 \pm 0.001$ & $0.973 \pm 0.002$ & $0.980 \pm 0.002$ \\
\hline$F_{\mathrm{c}}$ & $0.957 \pm 0.001$ & $0.956 \pm 0.001$ & $0.957 \pm 0.001$ & $0.975 \pm 0.001$ & $0.972 \pm 0.001$ & $1.000 \pm 0.003$ & $0.993 \pm 0.002$ \\
\hline$i\left[^{\circ}\right]$ & $87.96 \pm 0.04$ & $87.83 \pm 0.02$ & $87.78 \pm 0.04$ & $82.32 \pm 0.03$ & $82.05 \pm 0.02$ & $87.1 \pm 0.4$ & $87.3 \pm 0.3$ \\
\hline$\beta_{\mathrm{c}}$ & $0.088 \pm 0.004$ & $0.083 \pm 0.002$ & $0.067 \pm 0.003$ & $0.18 \pm 0.02$ & $0.17 \pm 0.01$ & $0.11 \pm 0.05$ & $0.10 \pm 0.03$ \\
\hline$c_{\mathrm{h}}$ & 0.120 & 0.087 & 0.053 & 0.009 & -0.004 & 0.082 & 0.068 \\
\hline$d_{\mathrm{h}}$ & 0.621 & 0.783 & 0.704 & 0.824 & 0.721 & 0.837 & 0.731 \\
\hline$a_{\mathrm{c}}$ & 0.874 & 0.852 & 0.799 & 0.836 & 0.764 & 0.849 & 0.781 \\
\hline$b_{\mathrm{c}}$ & -0.130 & 0.002 & 0.138 & 0.140 & 0.226 & 0.069 & 0.192 \\
\hline$\Omega_{\mathrm{h}}$ & 7.621 & 7.657 & 7.652 & 3.146 & 3.147 & 2.835 & 2.817 \\
\hline$\Omega_{\mathrm{c}}$ & 2.119 & 2.120 & 2.119 & 2.493 & 2.497 & 2.769 & 2.779 \\
\hline$R_{\mathrm{h}}[D=1]$ & 0.134 & 0.133 & 0.133 & 0.349 & 0.349 & 0.413 & 0.416 \\
\hline$R_{\mathrm{c}}[D=1]$ & 0.202 & 0.202 & 0.203 & 0.254 & 0.253 & 0.291 & 0.289 \\
\hline$L_{\mathrm{h}} /\left(L_{\mathrm{h}}+L_{\mathrm{c}}\right)$ & 0.919 & 0.889 & 0.800 & 0.950 & 0.921 & 0.948 & 0.914 \\
\hline $\mathcal{M}_{\mathrm{h}}\left[M_{\odot}\right]$ & 1.80 & 1.80 & 1.80 & 0.97 & 0.97 & 1.54 & 1.54 \\
\hline $\mathcal{M}_{\mathrm{c}}\left[M_{\odot}\right]$ & 0.26 & 0.26 & 0.26 & 0.29 & 0.29 & 0.69 & 0.69 \\
\hline $\mathcal{R}_{\mathrm{h}}\left[R_{\odot}\right]$ & 1.58 & 1.57 & 1.57 & 1.27 & 1.27 & 1.61 & 1.63 \\
\hline $\mathcal{R}_{\mathrm{c}}\left[R_{\odot}\right]$ & 2.54 & 2.54 & 2.54 & 0.96 & 0.95 & 1.15 & 1.14 \\
\hline $\log g_{\mathrm{h}}$ & 4.30 & 4.30 & 4.30 & 4.22 & 4.22 & 4.21 & 4.20 \\
\hline $\log g_{\mathrm{c}}$ & 3.04 & 3.04 & 3.04 & 3.94 & 3.94 & 4.15 & 4.16 \\
\hline$M_{\mathrm{bol}}^{\mathrm{h}}$ & 2.01 & 2.02 & 2.02 & 2.09 & 2.09 & 2.25 & 2.23 \\
\hline$M_{\mathrm{bol}}^{\mathrm{c}}$ & 3.26 & 3.30 & 3.35 & 4.90 & 4.96 & 4.80 & 4.77 \\
\hline$a_{\mathrm{orb}}\left[R_{\odot}\right]$ & 11.8 & 11.8 & 11.8 & 3.54 & 3.54 & 3.69 & 3.69 \\
\hline
\end{tabular}

BaSeL approximation of stellar atmosphere. $\left([\mathrm{Fe} / \mathrm{H}]_{\mathrm{h}, \mathrm{c}}=0.0\right.$ - adopted metallicity of the components).

Note: Same as in Table 1, except $a_{\mathrm{c}}, b_{\mathrm{c}}$ - nonlinear limb-darkening coefficients of the components (logarithmic law).

\section{5. $R Z$ Draconis $(P \sim 0 \mathrm{~d} .5509)$}

The $B V$ observations with normal points for this system were taken from Kreiner et al.'s (1994) paper. The value of the mass-ratio used in the light-curve analysis was $q=m_{\mathrm{c}} / m_{\mathrm{h}}=$ 0.445 , is the photometrically estimated one, and is taken from the same paper. As concerns the temperature of the primary component, we adopted the value of $T_{\mathrm{h}}=8150 \mathrm{~K}$, according to its spectral type: A5 V. The primary's gravity-darkening exponent was fixed to the value $\beta_{\mathrm{h}}=0.25$, and the albedos were taken to be equal to $A_{\mathrm{h}}=1.0$ and $A_{\mathrm{c}}=0.5$.

The results of our light-curve analysis for this system are presented in Table 2, and a graphic presentation is given in Fig. 5 (right). BaSeL approximation gives the best agreement between the individual $B$ and $V$ passband solutions. We found $\beta_{\mathrm{c}} \sim 0.105$ as the gravity-darkening exponent of the secondary, which is very close to the value expected for stars with convective envelopes, $\beta \sim 0.08$.

\subsection{TV Cassiopeiae $(P \sim 1$ d 8126$)$}

In the analysis of this system we used the $B V$ light curves with normal points, published in the Papoušek's (1974) paper. The light-curve analysis was made with the spectroscopically estimated mass-ratio of the components, $q_{\mathrm{sp}}=m_{\mathrm{c}} / m_{\mathrm{h}}=0.405$ (Khalesseh \& Hill 1992). The adopted value of the temperature of the primary was $T_{\mathrm{h}}=10500 \mathrm{~K}$ in agreement to its spectral type, B9V. A fixed value of the 

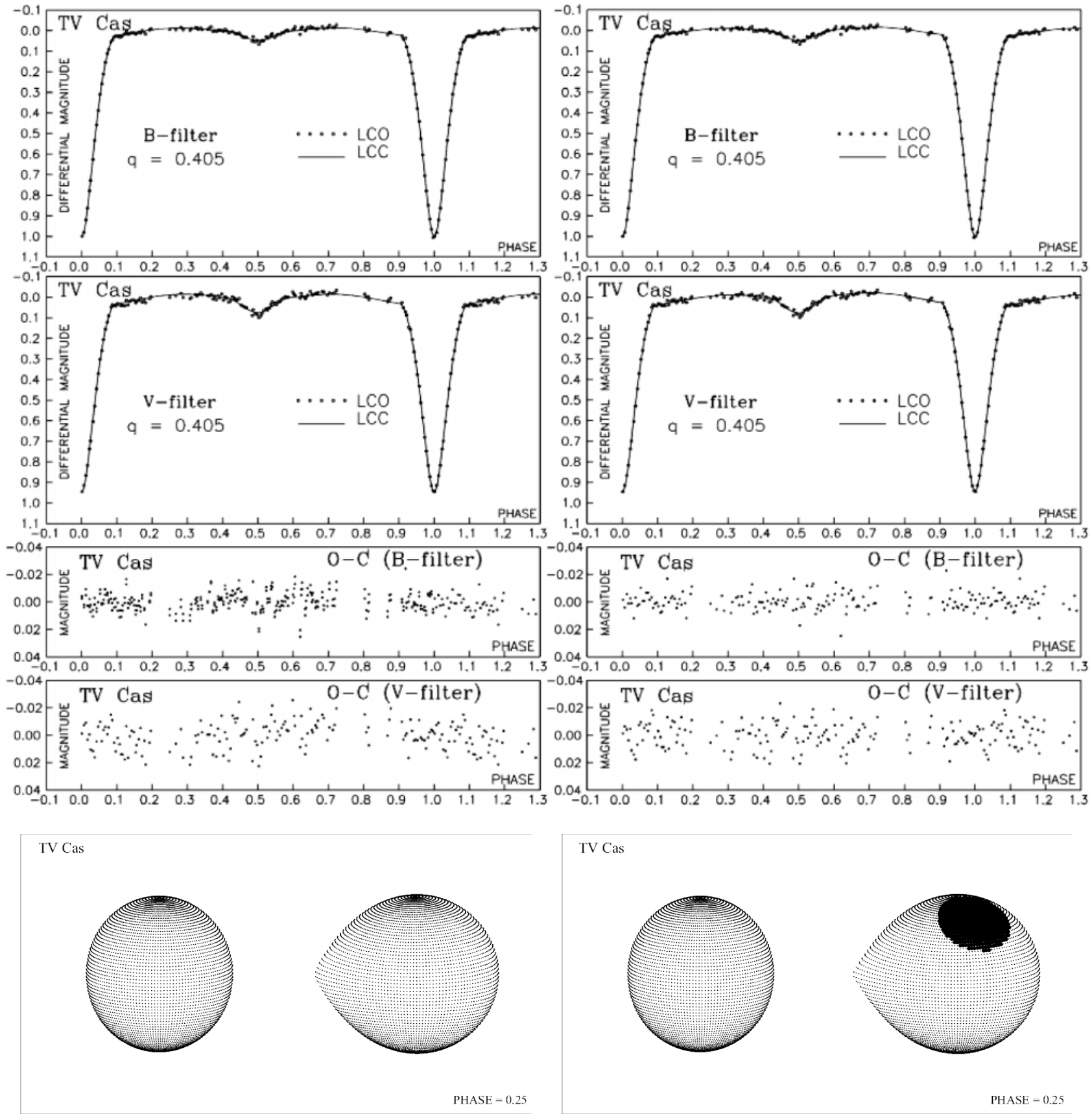

TV Cas
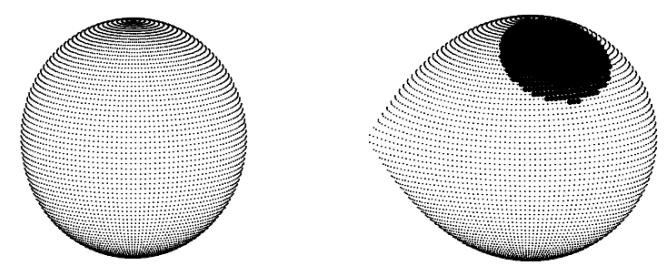

PHASE $=0.25$

Fig. 3. Observed (LCO) and final synthetic (LCC) light curves of TV Cas with final O-C residuals obtained by analysing $B$ and $V$ observations; The view of the system TV Cas at orbital phase 0.25 , obtained with the parameters estimated by analysing observations. Left - gravity-darkening exponent of the cooler secondary component is a free parameter; Right - supposing a spot on the secondary component.

gravity-darkening exponent for the primary $\left(\beta_{\mathrm{h}}=0.25\right)$ was used, and the adopted values of the albedos of the components were $A_{\mathrm{h}}=1.0$ and $A_{\mathrm{c}}=0.5$.

The numerical and graphical presentations of the results are given in Table 3 (left) and Fig. 3 (left). The best agreement between the individual $B$ and $V$ passband solutions was obtained with the BaSeL approximation. For the gravitydarkening exponent of the secondary we obtained $\beta_{\mathrm{c}} \sim 0.155$, which is almost $94 \%$ beyond the value expected for stars with convective envelopes $(\beta \sim 0.08)$. However, we have to say here that there was a certain degree of asymmetry present in the light curves noted in the different heights of the maxima
(Max $1<\operatorname{Max} 2$ ). This fact can be interpreted as a consequence of the mass transfer between the components of the system or by the spot activity on the convective secondary star. Tests made with both of these hypotheses yield to very interesting results: if we fix the gravity-darkening exponents for the primary and secondary to von Zeipel's and Lucy's values, respectively, the light curves can be well fitted by employing the Roche model taking into account the presence of a spotted area on the surface of the secondary star. In our programme these active regions are approximated by circular spots, characterised by the temperature contrast of the spot with respect to the surrounding photosphere $\left(A_{\mathrm{S}}=T_{\mathrm{S}} / T_{\mathrm{c}}\right)$, by the angular 
Table 3. Results of the analysis of TV Cas light curves obtained by solving the inverse problem for the Roche model. Left - Gravity-darkening exponent of the cooler secondary component $\left(\beta_{\mathrm{c}}\right)$ is a free parameter; Right - Spot on the secondary component.

\begin{tabular}{|c|c|c|c|c|}
\hline $\begin{array}{l}\text { CB system } \\
\text { quantity }\end{array}$ & $\begin{array}{l}\text { TV Cas } \\
B-\text { filter }\end{array}$ & $\begin{array}{l}\text { TV Cas } \\
V \text {-filter }\end{array}$ & $\begin{array}{l}\text { TV Cas } \\
B-\text { filter }\end{array}$ & $\begin{array}{l}\text { TV Cas } \\
V \text {-filter }\end{array}$ \\
\hline$n$ & 143 & 143 & 143 & 143 \\
\hline$\Sigma(\mathrm{O}-\mathrm{C})^{2}$ & 0.0570 & 0.1228 & 0.0503 & 0.0988 \\
\hline$\sigma$ & 0.0200 & 0.0294 & 0.0188 & 0.0264 \\
\hline$q=m_{\mathrm{c}} / m_{\mathrm{h}}$ & 0.405 & & & \\
\hline$T_{\mathrm{h}}$ & 10500 & & & \\
\hline$\beta_{\mathrm{h}}$ & 0.25 & & & \\
\hline$A_{\mathrm{h}}$ & 1.0 & & & \\
\hline$A_{\mathrm{c}}$ & 0.5 & & & \\
\hline$f_{\mathrm{h}}=f_{\mathrm{c}}$ & 1.0 & & & \\
\hline$T_{\mathrm{c}}$ & $5400 \pm 35$ & $5361 \pm 35$ & $5506 \pm 29$ & $5471 \pm 28$ \\
\hline$F_{\mathrm{h}}$ & $0.644 \pm 0.002$ & $0.643 \pm 0.002$ & $0.643 \pm 0.002$ & $0.642 \pm 0.003$ \\
\hline$F_{\mathrm{c}}$ & $0.999 \pm 0.001$ & $0.996 \pm 0.001$ & $1.000 \pm 0.001$ & $1.000 \pm 0.001$ \\
\hline$i\left[^{\circ}\right]$ & $79.01 \pm 0.02$ & $78.99 \pm 0.03$ & $79.04 \pm 0.03$ & $79.01 \pm 0.04$ \\
\hline$A_{\mathrm{S}}=T_{\mathrm{S}} / T_{\mathrm{c}}$ & & & $0.71 \pm 0.10$ & $0.69 \pm 0.09$ \\
\hline$\theta_{\mathrm{S}}\left[^{\circ}\right]$ & & & $28.5 \pm 2.9$ & $28.8 \pm 1.9$ \\
\hline$\lambda_{\mathrm{S}}\left[^{\circ}\right]$ & & & $60.3 \pm 11.9$ & $66.7 \pm 9.3$ \\
\hline$\varphi_{\mathrm{S}}\left[^{\circ}\right]$ & & & $55.7 \pm 8.7$ & $53.9 \pm 9.4$ \\
\hline$\beta_{\mathrm{c}}$ & $0.15 \pm 0.02$ & $0.16 \pm 0.02$ & $0.08^{*}$ & $0.08^{*}$ \\
\hline$c_{\mathrm{h}}$ & -0.045 & -0.041 & -0.045 & -0.041 \\
\hline$d_{\mathrm{h}}$ & 0.847 & 0.727 & 0.847 & 0.727 \\
\hline$a_{\mathrm{c}}$ & 0.847 & 0.783 & 0.844 & 0.777 \\
\hline$b_{\mathrm{c}}$ & 0.072 & 0.186 & 0.094 & 0.203 \\
\hline$\Omega_{\mathrm{h}}$ & 3.987 & 3.995 & 3.994 & 4.001 \\
\hline$\Omega_{\mathrm{c}}$ & 2.690 & 2.695 & 2.688 & 2.688 \\
\hline$R_{\mathrm{h}}[D=1]$ & 0.278 & 0.277 & 0.277 & 0.277 \\
\hline$R_{\mathrm{c}}[D=1]$ & 0.283 & 0.282 & 0.283 & 0.283 \\
\hline$L_{\mathrm{h}} /\left(L_{\mathrm{h}}+L_{\mathrm{c}}\right)$ & 0.946 & 0.900 & 0.942 & 0.895 \\
\hline $\mathcal{M}_{\mathrm{h}}\left[M_{\odot}\right]$ & 3.78 & 3.78 & 3.78 & 3.78 \\
\hline $\mathcal{M}_{\mathrm{c}}\left[M_{\odot}\right]$ & 1.53 & 1.53 & 1.53 & 1.53 \\
\hline $\mathcal{R}_{\mathrm{h}}\left[R_{\odot}\right]$ & 3.08 & 3.07 & 3.07 & 3.06 \\
\hline $\mathcal{R}_{\mathrm{c}}\left[R_{\odot}\right]$ & 3.30 & 3.29 & 3.31 & 3.31 \\
\hline $\log g_{\mathrm{h}}$ & 4.04 & 4.04 & 4.04 & 4.04 \\
\hline $\log g_{\mathrm{c}}$ & 3.58 & 3.59 & 3.58 & 3.58 \\
\hline$M_{\mathrm{bol}}^{\mathrm{h}}$ & -0.25 & -0.24 & -0.24 & -0.24 \\
\hline$M_{\mathrm{bol}}^{\mathrm{c}}$ & 2.49 & 2.53 & 2.40 & 2.43 \\
\hline$a_{\text {orb }}\left[R_{\odot}\right]$ & 10.90 & 10.90 & 10.90 & 10.90 \\
\hline
\end{tabular}

BaSeL approximation of stellar atmosphere $\left([\mathrm{Fe} / \mathrm{H}]_{\mathrm{h}, \mathrm{c}}=0.0-\right.$ adopted metallicity of the components).

Note: Same as in Table 1 , except $A_{\mathrm{S}}$ - spot temperature coefficient, $\theta_{\mathrm{S}}, \lambda_{\mathrm{S}}, \varphi_{\mathrm{S}}-$ spot radius, longitude and latitude (in arc degrees), $a_{\mathrm{c}}, b_{\mathrm{c}}-$ nonlinear limb-darkening coefficients of the components (logarithmic law).

dimension (radius) of the spot $\left(\theta_{\mathrm{S}}\right)$ and by the longitude $\left(\lambda_{\mathrm{S}}\right)$ and latitude $\left(\varphi_{\mathrm{S}}\right)$ of the spot's center. The longitude $\left(\lambda_{\mathrm{S}}\right)$ is measured clockwise (as viewed from the direction of the $+Z$-axis) from the $+X$-axis (line connecting star centers) in the range $0^{\circ}-$ $360^{\circ}$. The latitude $\left(\varphi_{\mathrm{S}}\right)$ is measured from $0^{\circ}$ at the stellar equator (orbital plane) to $+90^{\circ}$ towards the "north" $(+Z)$ and $-90^{\circ}$ towards the "south" $(-Z)$ pole. Table 3 (right) comprises the results obtained in such a case, and their graphical presentation is given in Fig. 3 (right). Figure 3 shows that in this case we have a better fit of the observed light curve. The light-curve asymmetry, possibly caused by a spotted area on the secondary, is relatively weakly expressed, which can be interpreted through a great difference between the temperatures of the components (see Table 3). A prevailing part of total luminosity comes from the primary (hot) component. So, the spotted area on the secondary (cool) component has a small but noticeable effect on the light-curve asymmetry. 

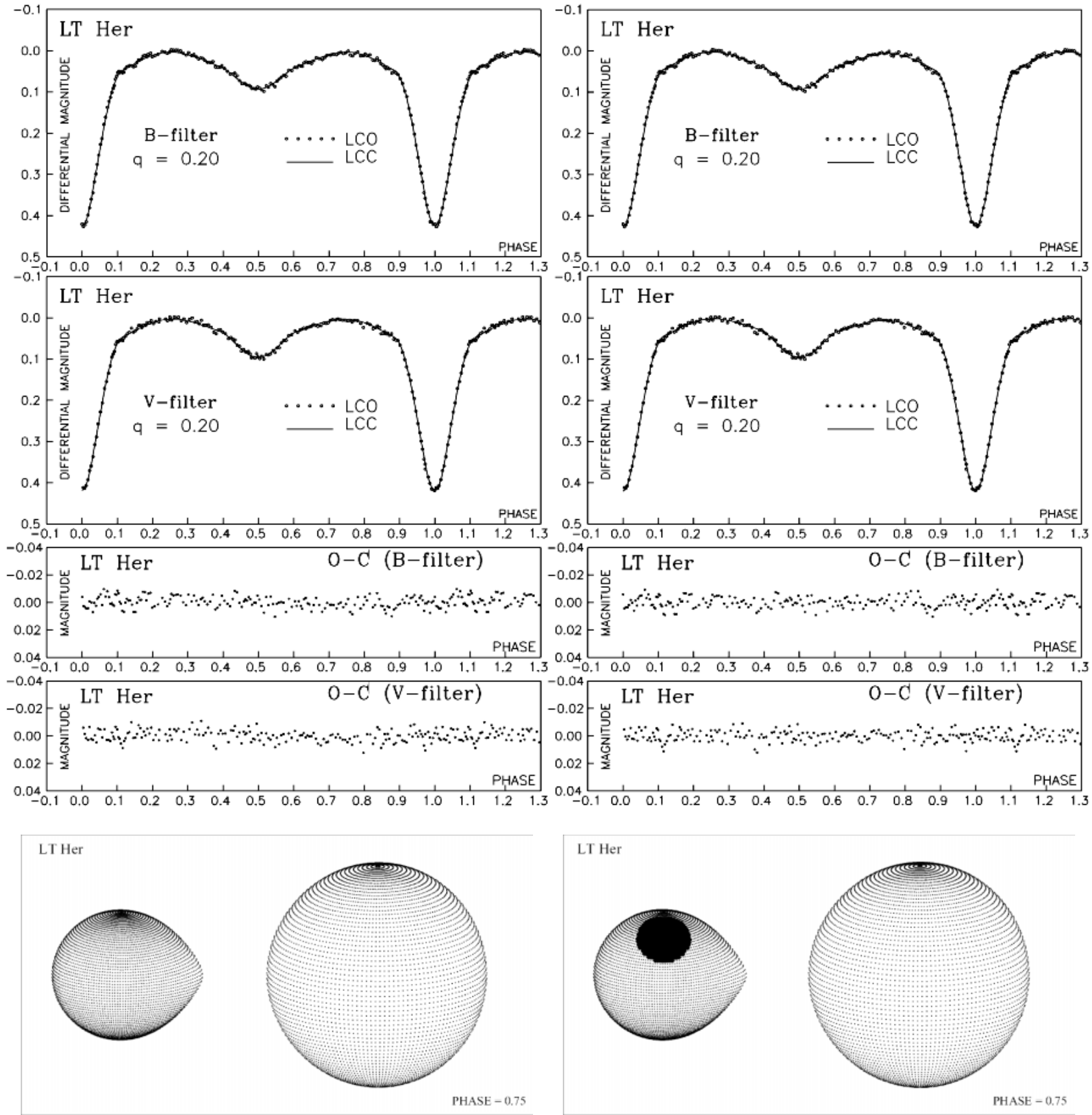

Fig. 4. Observed (LCO) and final synthetic (LCC) light curves of LT Her with final O-C residuals obtained by analysing the $B$ and $V$ observations and the view of the system LT Her at orbital phase 0.75 , obtained with the parameters estimated by analysing observations. Left gravity-darkening exponent of the cooler secondary component is a free parameter; Right - spot on the secondary component.

In this case the final $\mathrm{O}-\mathrm{C}$ residuals between observed (LCO) and final synthetic (LCC) light curves of TV Cas, obtained by analysing $B$ and $V$ observations, are much better (without asymmetry). We think that the existence of the spotted area on the secondary as the cause of the light-curve anomaly is more reasonable than the anomalous values of the gravitydarkening exponent.

\section{7. $L T$ Hercules $(P \sim 1$ d.084 $)$}

$B V$ photometric light curves for this eclipsing binary were published by Ebbighausen \& Penegor (1974). Following Russo \& Milano (1983), we fix the photometric mass-ratio to $q=m_{\mathrm{c}} / m_{\mathrm{h}}=0.20$, and the effective temperature of the primary component to $T_{\mathrm{h}}=9400 \mathrm{~K}$. On the basis of the spectral type of the components $(\mathrm{A} 2 \mathrm{~V}+[\mathrm{G} 3])$ the gravity-darkening exponent of the primary was fixed to $\beta_{\mathrm{h}}=0.25$, and for the albedos of the components we adopted the values $A_{\mathrm{h}}=1.0$ and $A_{\mathrm{c}}=0.5$, respectively.

The light-curve analysis of the LT Her, made within the Roche model, gave us the empirically estimated exponent of the gravity-darkening for the secondary component filling its Roche lobe. The gravity-darkening exponent of the primary component underfilling its Roche lobe was fixed to von Zeipel's value for stars in hydrostatic and radiative equilibrium. 
Table 4. Results of the analysis of LT Her light curves obtained by solving the inverse problem for the Roche model. Left - gravity-darkening exponent of the cooler secondary component $\left(\beta_{\mathrm{c}}\right)$ is a free parameter; Right - spot on the secondary component.

\begin{tabular}{|c|c|c|c|c|}
\hline $\begin{array}{l}\text { CB system } \\
\text { quantity }\end{array}$ & $\begin{array}{l}\text { LT Her } \\
B-\text { filter }\end{array}$ & $\begin{array}{l}\text { LT Her } \\
V \text {-filter }\end{array}$ & $\begin{array}{l}\text { LT Her } \\
B \text {-filter }\end{array}$ & $\begin{array}{l}\text { LT Her } \\
V \text {-filter }\end{array}$ \\
\hline$n$ & 176 & 171 & 176 & 171 \\
\hline$\Sigma(\mathrm{O}-\mathrm{C})^{2}$ & 0.0030 & 0.0031 & 0.0028 & 0.0027 \\
\hline$\sigma$ & 0.0042 & 0.0043 & 0.0040 & 0.0040 \\
\hline$q=m_{\mathrm{c}} / m_{\mathrm{h}}$ & 0.20 & & & \\
\hline$T_{\mathrm{h}}$ & 9400 & & & \\
\hline$\beta_{\mathrm{h}}$ & 0.25 & & & \\
\hline$A_{\mathrm{h}}$ & 1.0 & & & \\
\hline$A_{\mathrm{c}}$ & 0.5 & & & \\
\hline$f_{\mathrm{h}}=f_{\mathrm{c}}$ & 1.0 & & & \\
\hline$T_{\mathrm{c}}$ & $5474 \pm 46$ & $5291 \pm 43$ & $5453 \pm 44$ & $5266 \pm 40$ \\
\hline$F_{\mathrm{h}}$ & $0.821 \pm 0.002$ & $0.820 \pm 0.002$ & $0.821 \pm 0.002$ & $0.824 \pm 0.002$ \\
\hline$F_{\mathrm{c}}$ & $0.994 \pm 0.002$ & $0.993 \pm 0.002$ & $1.000 \pm 0.002$ & $0.993 \pm 0.002$ \\
\hline$i\left[^{\circ}\right]$ & $75.54 \pm 0.05$ & $75.58 \pm 0.05$ & $75.56 \pm 0.05$ & $75.57 \pm 0.06$ \\
\hline$A_{\mathrm{S}}=T_{\mathrm{S}} / T_{\mathrm{c}}$ & & & $0.80 \pm 0.09$ & $0.79 \pm 0.08$ \\
\hline$\theta_{\mathrm{S}}\left[^{\circ}\right]$ & & & $25.8 \pm 3.3$ & $25.1 \pm 3.0$ \\
\hline$\lambda_{\mathrm{S}}\left[^{\circ}\right]$ & & & $259.9 \pm 18.8$ & $280.1 \pm 15.6$ \\
\hline$\varphi_{\mathrm{S}}\left[{ }^{\circ}\right]$ & & & $49.7 \pm 11.6$ & $51.4 \pm 15.3$ \\
\hline$\beta_{\mathrm{c}}$ & $0.26 \pm 0.03$ & $0.27 \pm 0.04$ & $0.08^{*}$ & $0.08^{*}$ \\
\hline$c_{\mathrm{h}}$ & 0.022 & 0.003 & 0.022 & 0.003 \\
\hline$d_{\mathrm{h}}$ & 0.819 & 0.720 & 0.819 & 0.720 \\
\hline$a_{\mathrm{c}}$ & 0.847 & 0.788 & 0.848 & 0.789 \\
\hline$b_{\mathrm{c}}$ & 0.092 & 0.167 & 0.087 & 0.163 \\
\hline$\Omega_{\mathrm{h}}$ & 2.686 & 2.689 & 2.686 & 2.677 \\
\hline$\Omega_{\mathrm{c}}$ & 2.238 & 2.239 & 2.233 & 2.240 \\
\hline$R_{\mathrm{h}}[D=1]$ & 0.400 & 0.399 & 0.400 & 0.401 \\
\hline$R_{\mathrm{c}}[D=1]$ & 0.232 & 0.231 & 0.233 & 0.231 \\
\hline$L_{\mathrm{h}} /\left(L_{\mathrm{h}}+L_{\mathrm{c}}\right)$ & 0.964 & 0.954 & 0.965 & 0.957 \\
\hline
\end{tabular}

Black-body approximation of stellar atmosphere.

Note: Same as in Table 1, except $A_{\mathrm{S}}$ - spot temperature coefficient, $\theta_{\mathrm{S}}, \lambda_{\mathrm{S}}, \varphi_{\mathrm{S}}-$ spot radius, longitude and latitude (in arc degrees), $a_{\mathrm{c}}, b_{\mathrm{c}}-$ nonlinear limb-darkening coefficients of the components (logarithmic law).

The best agreement between the individual $B$ and $V$ passband solutions, in the LT Her case, was obtained with the simple black-body approximation.

The results of the analysis are given in Table 4 (left) and their graphical presentation is shown in Fig. 4 (left). For the gravity-darkening exponent of the secondary we found the value $\beta_{\mathrm{c}} \sim 0.265$, which is well beyond - being ( $\sim 3.3$ times) higher - than the value expected for stars with convective envelopes $(\beta \sim 0.08)$. But, it has to be said that our estimated value of the gravity-darkening exponent is significantly lower than that found by others (Kitamura \& Nakamura 1987b), which is about 5.5 times higher.

The estimated high value of the gravity-darkening exponent of LT Her can be overcome, if we assume a possible solar type spot activity, as we did in the TV Cas case. Thus, working in a similar way, fixing the gravity-darkening exponents for the primary and secondary stars to von Zeipel's and Lucy's values, respectively, the light curves can be well fitted by employing the Roche model taking into account the presence of a spotted area on the secondary. Table 4 (right) comprises the results obtained in such a case, and their graphical presentation is given in Fig. 4 (right). From results in Table 4, and Fig. 4, we can see that in this case we have a better fit. The spotted area on the secondary causes a very small light-curve asymmetry. This can be interpreted as a consequence of the great difference between the temperatures of the components. In total luminosity of this system, the primary (hot) component contributes with $\sim 96 \%$, and the spotted area on the secondary (cool) component has a small effect on the light-curve asymmetry.

\section{8. $Z Z$ Crucis $(P \sim 1$ d. 862$)$}

The observations (normal points), published in Horak et al.'s (1999) paper, and carried out between 1965 and 1968 with the use of Walraven $U L B V$ filters $(U-367 \mathrm{~nm}, L-390 \mathrm{~nm}, B-$ $429.5 \mathrm{~nm}$ and $V-545 \mathrm{~nm}$ ), were used in the present analysis. The difficult modelling of Balmer discontinuity forced us to use only $B$ and $V$ observations in the present light-curve 
Table 5. Results of the analysis of ZZ Cru and W UMi light curves obtained by solving the inverse problem for the Roche model. Gravitydarkening exponent of the cooler secondary component $\left(\beta_{\mathrm{c}}\right)$ is a free parameter.

\begin{tabular}{|c|c|c|c|c|c|}
\hline $\begin{array}{l}\text { CB system } \\
\text { quantity }\end{array}$ & $\begin{array}{l}\text { ZZ Cru } \\
B \text {-filter }\end{array}$ & $\begin{array}{l}\text { ZZ Cru } \\
V \text {-filter }\end{array}$ & $\begin{array}{l}\text { CB system } \\
\text { quantity }\end{array}$ & $\begin{array}{l}\text { W UMi } \\
B \text {-filter }\end{array}$ & $\begin{array}{l}\text { W UMi } \\
V \text {-filter }\end{array}$ \\
\hline$n$ & 96 & 96 & $n$ & 312 & 312 \\
\hline$\Sigma(\mathrm{O}-\mathrm{C})^{2}$ & 0.1564 & 0.1326 & $\Sigma(\mathrm{O}-\mathrm{C})^{2}$ & 0.0377 & 0.0232 \\
\hline$\sigma$ & 0.0406 & 0.0374 & $\sigma$ & 0.0110 & 0.0086 \\
\hline$q=m_{\mathrm{c}} / m_{\mathrm{h}}$ & 0.25 & & $q=m_{\mathrm{c}} / m_{\mathrm{h}}$ & 0.48 & \\
\hline$T_{\mathrm{h}}$ & 11170 & & $T_{\mathrm{h}}$ & 9230 & \\
\hline$\beta_{\mathrm{h}}$ & 0.25 & & $\beta_{\mathrm{h}}$ & 0.25 & \\
\hline$A_{\mathrm{h}}$ & 1.0 & & $A_{\mathrm{h}}$ & 1.0 & \\
\hline$A_{\mathrm{c}}$ & 1.0 & & $A_{\mathrm{c}}$ & 0.5 & \\
\hline$f_{\mathrm{h}}=f_{\mathrm{c}}$ & 1.0 & & $f_{\mathrm{h}}=f_{\mathrm{c}}$ & 1.0 & \\
\hline$T_{\mathrm{c}}$ & $8683 \pm 35$ & $8425 \pm 35$ & $T_{\mathrm{c}}$ & $5416 \pm 37$ & $5400 \pm 21$ \\
\hline$F_{\mathrm{h}}$ & $0.723 \pm 0.004$ & $0.721 \pm 0.004$ & $F_{\mathrm{h}}$ & $0.829 \pm 0.002$ & $0.828 \pm 0.002$ \\
\hline$F_{\mathrm{c}}$ & $1.000 \pm 0.002$ & $1.000 \pm 0.002$ & $F_{\mathrm{c}}$ & $0.995 \pm 0.001$ & $0.995 \pm 0.001$ \\
\hline$i\left[^{\circ}\right]$ & $82.4 \pm 0.1$ & $82.4 \pm 0.1$ & $i$ & $82.41 \pm 0.04$ & $82.46 \pm 0.04$ \\
\hline$\beta_{\mathrm{c}}$ & $0.25 \pm 0.02$ & $0.25 \pm 0.02$ & $\beta_{\mathrm{c}}$ & $0.08 \pm 0.03$ & $0.08 \pm 0.02$ \\
\hline$c_{\mathrm{h}}$ & -0.068 & -0.055 & $c_{\mathrm{h}}$ & 0.037 & 0.014 \\
\hline$d_{\mathrm{h}}$ & 0.843 & 0.719 & $d_{\mathrm{h}}$ & 0.811 & 0.716 \\
\hline$c_{\mathrm{c}}$ & 0.093 & 0.064 & $a_{\mathrm{c}}$ & 0.848 & 0.783 \\
\hline$d_{\mathrm{c}}$ & 0.781 & 0.715 & $b_{\mathrm{c}}$ & 0.080 & 0.184 \\
\hline$\Omega_{\mathrm{h}}$ & 3.177 & 3.187 & $\Omega_{\mathrm{h}}$ & 3.342 & 3.345 \\
\hline$\Omega_{\mathrm{c}}$ & 2.353 & 2.353 & $\Omega_{\mathrm{c}}$ & 2.846 & 2.845 \\
\hline$R_{\mathrm{h}}[D=1]$ & 0.340 & 0.339 & $R_{\mathrm{h}}[D=1]$ & 0.346 & 0.346 \\
\hline$R_{\mathrm{c}}[D=1]$ & 0.248 & 0.248 & $R_{\mathrm{c}}[D=1]$ & 0.295 & 0.295 \\
\hline$L_{\mathrm{h}} /\left(L_{\mathrm{h}}+L_{\mathrm{c}}\right)$ & 0.767 & 0.756 & $L_{\mathrm{h}} /\left(L_{\mathrm{h}}+L_{\mathrm{c}}\right)$ & 0.947 & 0.908 \\
\hline
\end{tabular}

ZZ Cru: Carbon \& Gingerich (1969) approximation of stellar atmosphere.

W UMin: BaSeL approximation of stellar atmosphere $\left([\mathrm{Fe} / \mathrm{H}]_{\mathrm{h}, \mathrm{c}}=0.0\right.$ - adopted metallicity of the components).

Note: Same as in Table 1, except $a_{\mathrm{c}}, b_{\mathrm{c}}$ - nonlinear limb-darkening coefficients of the components (logarithmic law).

analysis. The fixed, photometrically estimated value of the mass-ratio of the components was $q=m_{\mathrm{c}} / m_{\mathrm{h}}=0.25$ (Horak et al. 1999). Based on the spectral type of the primary (B8) its temperature was taken to be $T_{\mathrm{h}}=11170 \mathrm{~K}$. The gravitydarkening exponent of the secondary was fixed to the value $\beta_{\mathrm{h}}=0.25$, and the values adopted for the albedos of the components were $A_{\mathrm{h}}=A_{\mathrm{c}}=1.0$.

The results of the light-curve analysis are given in Table 5 (CG approximation) and their graphic presentation is in Fig. 6 (left). We obtained the value $\beta_{\mathrm{c}} \sim 0.25$ for the gravity-darkening exponent of the secondary, which is the expected value for stars in hydrostatic and radiative equilibrium.

\section{9. $W$ Ursae Minoris $(P \sim 1.770156)$}

To analyse this system we used two colour ( $B$ and $V$ ) curves by Devinney et al. (1970). The initial values of the parameters of the model were taken according to the results of the analysis presented in the paper by Mardirossian et al. (1980). The temperature of the hotter primary was taken to be $T_{\mathrm{h}}=9230 \mathrm{~K}$, as suggested by Devinney et al. (1970), in agreement to its spectral classification of $\mathrm{A} 2 \pm 3$. Based on the spectral type of the components (A2+[G9IV]) the gravity-darkening exponent of the primary was fixed to $\beta_{\mathrm{h}}=0.25$, and the albedos of the components were taken as equal to $A_{\mathrm{h}}=1.0$ and $A_{\mathrm{c}}=0.5$. For the flux distribution we used the BaSeL approximation, which gives the best agreement between individual $(B$ and $V$ ) photometric solutions. The parameters of the system were estimated in the light-curve analysis with the mean photometrically estimated mass ratio $q=m_{\mathrm{c}} / m_{\mathrm{h}}=0.48 \pm 0.01$. The same value was estimated by Mardirossian et al. (1980). The obtained results shows that the cooler subgiant is in contact with its Roche lobe, while the filling factor for the hotter primary is $F_{\mathrm{h}} \sim 0.86$.

The results of the light-curve analysis are given in Table 5 and their graphical presentation is given in Fig. 6 (right). For the gravity-darkening exponent of the secondary we found the value $\beta_{\mathrm{c}} \sim 0.08$, which is the value expected for stars with convective envelopes.

\section{Discussion and conclusions}

The light curve analyses of nine semi-detached binary systems, presented in this paper, made within the Roche geometry, allowed us to estimate the gravity-darkening exponents of the secondary components filling their Roche lobes. The 

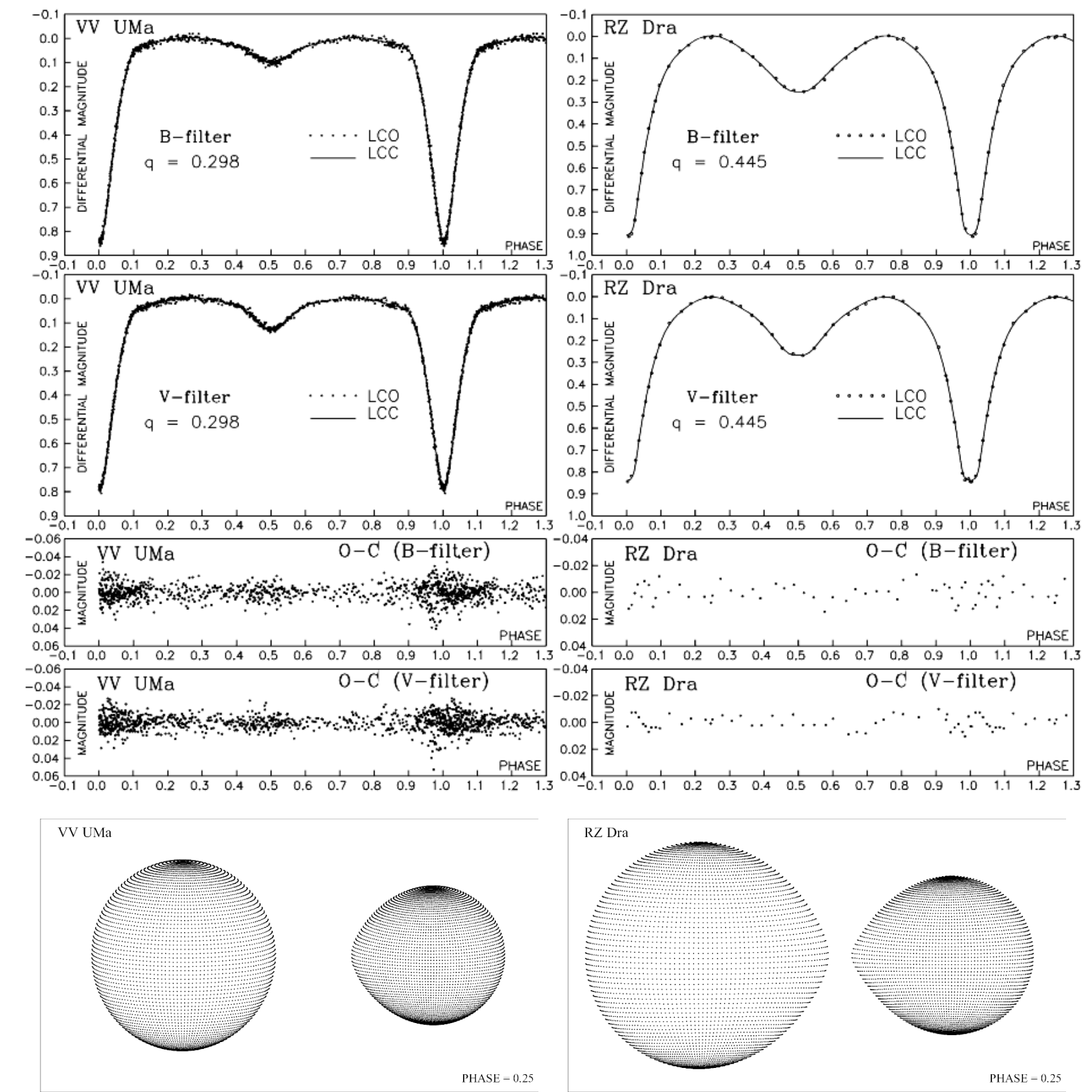

Fig. 5. Observed (LCO) and final synthetic (LCC) light curves of VV UMa and RZ Dra with final O-C residuals obtained by analysing $B$ and $V$ observations and the view of the systems at orbital phase 0.25 , obtained with the parameters estimated by analysing observations.

gravity-darkening exponents of the components underfilling their Roche lobes have been fixed to their theoretical values. That is to $\beta=0.25$ for stars in hydrostatic and radiative equilibrium, according to von Zeipel's (1924) value, and to $\beta=0.08$ for stars with convective envelopes, according to Lucy's (1967) calculations.

The values of the gravity-darkening exponents estimated in the light-curve analysis were compared to theoretical predictions obtained on the basis of Claret's (1998) tables. By interpolating the data from Claret's tables we obtained the tracks for corresponding observed masses of the secondary system components. These tracks are represented in Fig. 7 together with the semi-empirical values of the GDE's estimated from the lightcurve analysis.

Our results show that, in general, the estimated values are in close agreement to the theoretically predicted, with a few exceptions.

To be more specific:

A. For 4 binaries (ZZ Cru, RZ Dra, XZ Sgr and W UMi), there is a very good agreement between empirically estimated values of the gravity-darkening exponent and the theoretically predicted values.

B. For two of the systems, namely TV Cas \& LT Her, results are fine if spot activity is assumed. 

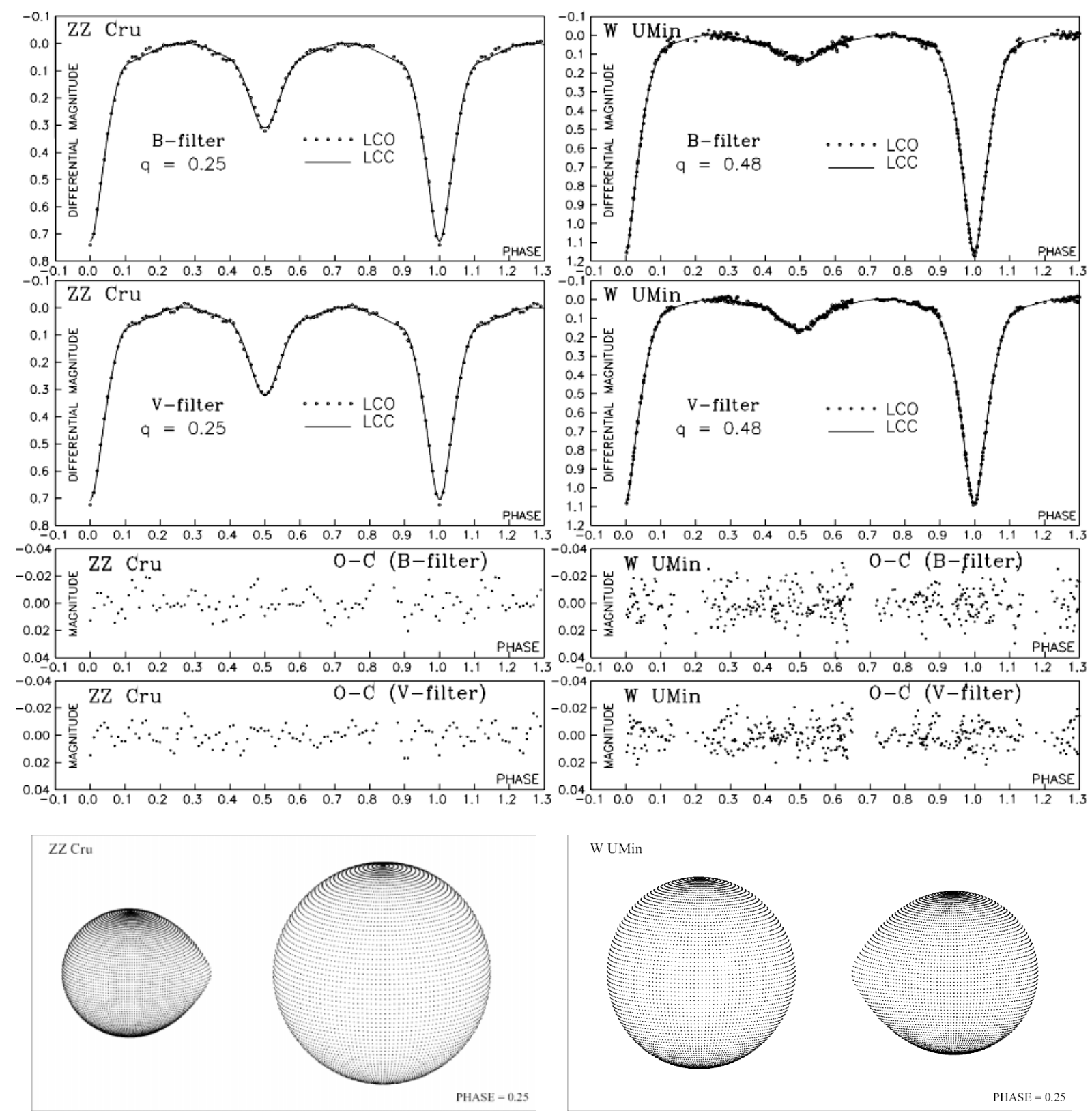

Fig. 6. Observed (LCO) and final synthetic (LCC) light curves of ZZ Cru and W UMi with final O-C residuals obtained by analysing $B$ and $V$ observations and the view of the the systems at orbital phase 0.25 , obtained with the parameters estimated by analysing observations.

C. And only for 3 systems (TT Aur, V Pup, and VV UMa) the empirically estimated values of the gravity-darkening exponent are higher than the values following from existing theories by von Zeipel and Lucy for radiative (hot star) and convective (cool star) envelopes, which are contained in the Claret's tables.

To these two basic but "old" theoretical expectations, new findings have been added. It has been found e.g. that the gravity-darkening phenomenon is not related only with the atmospheric parameters but it is also affected by the internal stellar structure and by the details of the rotation law (Claret 2000). The old values of 0.32 and 1.0 for convective and radiative envelopes, respectively are superseded by Claret's (1998) calculations where a smooth transition is achieved between both energy transport mechanisms. Actually the processes of transport energy can even exist simultaneously in a determined stellar envelope. The influence of the chemical composition on the gravity-darkening exponent is rated as slight and located mainly in the zone of the radiative/convective phase transition.

For the two massive B-type semi-detached systems, TT Aur and V Pup, the estimated values of the gravity-darkening exponents, (that found to have a mean value of 0.37 and 0.38 , respectively), are approximately 1.5 times higher than the theoretically predicted by von Zeipel (1924) for stars in radiative and hydrostatic equilibrium. Budding \& Kopal (1970) have also estimated a large value for the gravity-darkening of Algol itself, which was found to be 3.8 times greater than its theoretical value. Smith \& Worley (1974), and Smith (1975), have pointed out that von Zeipel gravity-darkening law is valid only under certain assumptions. Thus, it could be that anomalous 


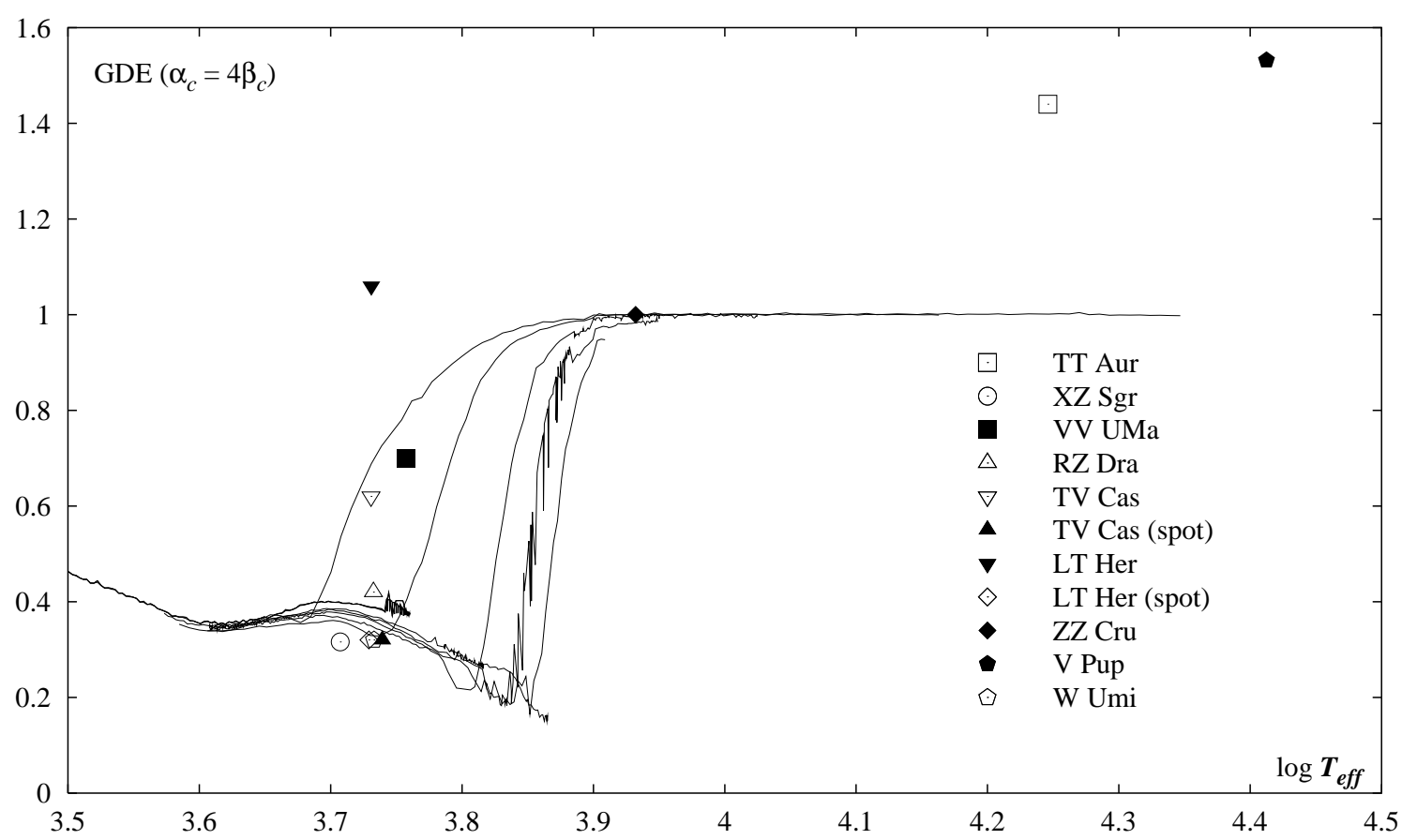

Fig. 7. Empirical gravity-darkening exponents (GDE) values and Claret's (1998) theoretical predictions as a function of effective temperature. The lines represent the tracks for corresponding observed masses of the secondary system components.

values of the gravity-darkening exponents obtained in these particular cases are the consequences of the simplified model not including the effects of the complex physics involved in this kind of systems. Unno et al. (1994) interpreted extremely large values of gravity-darkening exponents as a consequence of the enthalpy transport associated with the mass-loss from the secondary. It is reasonable to assume a model in which the less-massive secondary fills its Roche lobe and loses its mass towards the primary situated deeply within its own Roche lobe. At the place where the gas stream from the secondary hits the primary an active hot-spot region is formed. Moreover, via the reflection effect the hot-spot leads to an increase of the temperature on the secondary thus forming a bright "reflection cap" on its side against the hot-spot. Because of the gas stream between the components the shape of the light curve in semidetached systems can be affected by the presence of nonphotospheric matter. In the systems containing hot $\mathrm{O}$ and B-type components we should have in mind the effect of the radiation pressure on the shape of the equipotential surfaces (Djurašević 1986), changing also the positions of the Lagrangean points. This affects the ratio of radiative to gravitational forces which rapidly increases with $T_{\mathrm{eff}}^{4}$. The contribution of the radiative pressure in the total potential plays an important role leading by that to considerable deviations of the stellar surfaces from the assumed classical Roche geometry. Naturally, this affects the shape of the light curve, too (Drechsel et al. 1995). Taking into account the mentioned effect in the model for light-curve synthesis should make the empirically estimated values of the gravity-darkening exponents in the radiation pressure-modified case closer to the values following from von Zeipel's law. We think that such a model based on radiation pressure-modified Roche equipotentials (Drechsel et al. 1995) is more suitable for hot semi-detached systems than the classical Roche model in which the gravity-darkening exponent is treated as a free parameter in the inverse problem of the light-curve analysis. Moreover, Claret (2000) shows that the gravity-darkening exponent depends on the angular velocity distribution, on the opacity law and on the star's differential rotation. His paper suggests the effect of differential rotation as a possible cause of the anomalous gravity-darkening exponents within early-type systems.

Thus, we think that for the two early type systems, TT Aur \& V Pup of the present study, both mechanisms (differential rotation and radiative pressure effects) could be responsible for the higher derived values of GDE.

Within systems in which the cooler secondary component has a convective envelope (late spectral type-G,K), and if the light-curve shape allows it, it is reasonable to assume a possible solar type spot activity. If we fix the gravity-darkening exponent in these cases according to Lucy's (1967) calculations $(\beta \sim 0.08)$, the light curves can be well fitted by employing the Roche model which takes into account the presence of spots on the secondary star. This was proven for the CB systems TV Cas and LT Her. In these two cases, the treatment of the gravity-darkening exponent as a free parameter gave values that are higher than expected according to Lucy's (1967) or Claret's (1998) predictions. But if we fix the gravity-darkening exponent according to Lucy's (1967) calculations $(\beta \sim 0.08)$, the light curve anomalies are better fitted when a spotted area on the secondary component is assumed. This result confirms to a high degree the validity of theoretical predictions.

To estimate the gravity-darkening exponents, in some cases we used the same observations and the same input physical parameters as Kitamura \& Nakamura (1987b). Compared to their results, our analysis gave significantly lower values of the gravity-darkening exponents for these stars in general. Since 
we applied the Roche model and an adequate method of optimisation, enabling a simultaneous evaluation of the parameters of the system and gravity-darkening exponent, we think that these drastic differences can be entirely and directly attributed to the model and method used by those authors. The application of the Roche model yields to values that are closer to those expected according to existing theories by von Zeipel and Lucy for radiative (hot star) and convective (cool star) envelopes.

As concerns the VV UMa case, we estimated a mean GDE value of 0.165 , from the light-curve analysis of two color $(B$ and $V$ ) observations of Broglia \& Conconi (1977). The estimated GDE value, although almost double than expected for stars with convective envelopes $(\beta \sim 0.08)$, it is much lower than that estimated by others using the same data. This system consists a very interesting case. According to the new findings, based on the simultaneous four-color photometry $u v b y$, its primary component is a low-amplitude pulsating star (Lazaro et al. 2002). But, the light-curve analysis of these new observations does not indicate any anomaly of the gravity-darkening exponent of both system components. Moreover, the classical values of $\alpha_{\mathrm{h}}=4 \beta_{\mathrm{h}}=1$ and $\alpha_{\mathrm{c}}=4 \beta_{\mathrm{c}}=0.3$ computed by Claret method are quite adequate. These results suggest that our estimated higher value may be come from the old data used.

Acknowledgements. This work has been supported by the Athens University, (grant No. 70/4/3305), and by the Ministry for Sciences and Technology of Serbia through the project 1191 "Stellar physics". The authors would like to thank Dr. Claret for many useful comments and suggestions that helped us to improve the quality of our paper and for kindly giving the CD ROM with the necessary data at our disposal.

\section{References}

Al-Naimiy, H. M. 1978, Ap\&SS, 63, 181

Alencar, S. H. P., \& Vaz, L. P. R. 1997, A\&A, 326, 257

Andersen, J., Clausen, J. V., Giménez, A. \& Nordstström, B. 1983, A\&A, 128, 17

Anderson, L., \& Shu, F. H. 1977, ApJ, 214, 798

Bell, S. A., Adamson, A. J., \& Hilditch, R. W. 1987, MNRAS, 224, 649

Broglia, P., \& Conconi, P. 1977, A\&AS, 27, 285

Budding, E., \& Kopal, Z. 1970, Ap\&SS, 9, 343

Carbon, D., \& Gingerich, O. 1969, in Theory and Observation of Normal Stellar Atmospheres, ed. O. Gingerich (Cambridge: MIT Press), 377

Claret, A. 1995, A\&AS, 109, 441

Claret, A. 1998, A\&AS, 131, 395

Claret, A. 2000, A\&A, 359, 289

Clausen, J. V., Nordstström, B., \& Reipurth, B. 1983, A\&AS, 52, 323

Devinney, E. J., Hall, D. S., \& Ward, D. H. 1970, PASP, 82, 10

Díaz-Cordovés, J., Claret, A., \& Giménez, A. 1995, A\&AS, 110, 329

Djurašević, G. 1986, Ap\&SS, 124, 5

Djurašević, G. 1992, Ap\&SS, 197, 17

Djurašević, G., Zakirov, M., Hojaev, A., \& Arzumanyants, G. 1998, A\&AS, 131, 17

Djurašević, G., Rovithis-Livaniou, H., Rovithis, P., Georgiades, N., \& Erkapić, S. 2000, 20th SPIG, ed. Z. Lj. Petrović, M. M. Kuraica, N. Bibić, \& G. Malović, 501

Drechsel, H., Hass, S., Lorenz, R., \& Gayler, S. 1995, A\&A, 294, 723
Eaton, J. A., Wu, C.-C., \& Rucinski, S. M. 1980, ApJ, 239, 919

Ebbighausen, E. G., \& Penegor, G. 1974, PASP, 86, 203

Hilditch, R. W. 1981, MNRAS, 196, 305

Hill, G., Hilditch, R. W., Younger, F., \& Fisher, W. A. 1975, MmRAS, 79, 131

Horak, T. B., Grygar, J., van Houten, C. J., Chochol, D., \& Pribulla, T. 1999, Contr. Astron. Obs. Skalnate Pleso, 29, 127

Kappelmann, N., \& Walter, K. 1979, A\&AS, 38, 161

Khalesseh, B., \& Hill, G. 1992, A\&A, 257, 199

Kitamura, M., Tanabe, H., \& Nakamura, T. 1957, Astron. Soc. Jpn., 9 , 120

Kitamura, M., \& Nakamura, Y. 1983, Ann. Tokyo Astron. Obs., 2nd Ser., 19, 413

Kitamura, M., \& Nakamura, Y. 1986, Ann. Tokyo Astron. Obs., 2nd Ser., 21, 229

Kitamura, M., \& Nakamura, Y. 1987a, Ann. Tokyo Astron. Obs., 2nd Ser., 21, 311

Kitamura, M., \& Nakamura, Y. 1987b, Ann. Tokyo Astron. Obs., 2nd Ser., 21, 387

Kitamura, M., \& Nakamura, Y. 1988a, Ap\&SS, 145, 117

Kitamura, M., \& Nakamura, Y. 1988b, Ann. Tokyo Astron. Obs., 2nd Ser., 22, 31

Kitamura, M., \& Nakamura, Y. 1989, Publ. Nat. Astron. Obs. Jpn., 1, 43

Knipe, G. F. G. 1974, MNRAS, 167, 369

Kopal, Z. 1968, Ap\&SS, 2, 23

Kopal, Z., \& Kitamura, M. 1968, Advance in A\&A 6 (New York: Academic Press), 125

Kreiner, J. M., Pajdosz, G., Tremko, J., \& Zola, S. 1994, A\&A, 285, 459

Lázaro, C., Arévalo, M. J., Claret, A., Rodríguez, E., \& Olivares, I. 2001, MNRAS, 325, 617

Lejeune, T., Cuisinier, F., \& Buser, R. 1997, A\&AS, 125, 229

Lejeune, T., Cuisinier, F., \& Buser, R. 1998, A\&AS, 130, 65

Lucy, L. B. 1967, Zs. f. Ap., 65, 89

Mardirossian, F., Mezzetti, M., Predolin, F., \& Giuricin, G. 1980, A\&AS, 40, 57

Marquardt, D. W. 1963, J. Soc. Ind. Appl. Math., 11, 431

Nakamura, Y., \& Kitamura, M. 1992, Ap\&SS, 191, 267

Papoušek, J. 1974, BAICz, 25, 152

Press, W. H., Teukolsky, S. A., Vetterling, W. T., \& Flannery, B. P. 1992, Numerical Recipes in Fortran, The Art of Scientific Computing, 2nd edition (New York: Cambridge University Press), 120

Rafert, J. B. 1990, AJ, 100, 1253

Rafert, J. B., \& Twigg, L. W. 1980, MNRAS, 193, 79

Rovithis-Livaniou, H., Djurašević, G., Rovithis, P., Georgiades, N., \& Erkapić, S. 2001, 5th HelAsEt Meeting

Rucinski, S. M. 1969, Acta Astr., 19, 245

Russo, G., \& Milano, L. 1983, A\&AS, 69, 261

Sarna, M. J. 1989, A\&A, 224, 98

Smak, J. 1965, Acta Astr., 15, 327

Smith, R. C., \& Worley, R. 1974, MNRAS, 167, 199

Smith, R. C. 1975, MNRAS, 173, 97

Unno, W., Kiguchi, M., \& Kitamura, M. 1994, PASJ, 46, 613

Van Hamme, W. 1993, AJ, 106, 2096

Wachmann, A. A., Popper, D. M., \& Clausen, J. V. 1986, A\&A, 162, 62

Webbing, R. F. 1976, ApJ, 209, 829

Wilson, R. E., \& Devinney, E. J. 1971, ApJ, 166, 605

Zeipel, H. V. 1924, MNRAS, 84, 702 